\title{
Multimedia Content Description using Semantic Web Languages
}

\author{
Roberto García ${ }^{1}$, Chrisa Tsinaraki ${ }^{2}$, Òscar Celma ${ }^{3}$ and Stavros Christodoulakis ${ }^{2}$ \\ ${ }^{1}$ Human-Computer Interaction Research Group (GRIHO), Universitat de Lleida, Spain \\ rgarcia@diei.udl.cat \\ 2 Laboratory of Distributed Multimedia Information Systems and Applications (TUC/MUSIC), Technical University of \\ Crete, Chania, Crete, Greece, \{chrisa, stavros\}@ced.tuc.gr \\ 2 Music Technology Group, Universitat Pompeu Fabra, Spain \\ oscar.celma@iua.upf.edu
}

\section{Introduction}

During the last decades, digital media have revolutionised media reproduction. The availability of cheap consumer electronic devices that allow the consumption and management of digital multimedia content (e.g. MP3 players, digital cameras, DV camcorders, smart phones etc) has caused a media availability explosion. The amount of digital media that has been generated and stored, and which continues to do so at an exponential rate, has already become unmanageable without fine-grained computerised support.

This, in combination with the media distribution break-up carried out by the World Wide Web and the emergence of advanced network infrastructures that allow for the fast, efficient and reliable transmission of multimedia content, have formed an open multimedia consumption environment. Digital multimedia content services are provided in this environment, which offer high content quality, advanced interaction capabilities, media personalization and adaptation according to the user preferences and access conditions. Such an open environment will be successful only if it is based on standards that allow the services provided by different vendors to interoperate. The specification of different multimedia content description standards poses interoperability requirements and necessitates guidelines for semantic interoperability. These issues are discussed in detail in (Tzouvaras, Pan 2007).

The dominant standard in multimedia content description is the MPEG-7 (ISO MPEG Group), which provides rich general purpose multimedia content description capabilities, including both low-level features and high-level semantic description constructs. However, the lack of formal semantics in MPEG-7 makes the gap between low and high-level descriptions difficult to cope with for the existing tools. Consequently, low level features are common, as they can be easily extracted from the content, but there is a lack of high-level descriptions.

Low-level approaches, based on signal analysis, are proving to be extremely limiting in making multimedia database systems accessible and useful to the end-users. These content-based descriptors lay far away from what the users recognise as media description means (Celma, 2005). Consequently, recent research has begun to focus on bridging the semantic and conceptual gap that exists between the user and the computer from content-based to high-level descriptions. One approach to overcome this gap is the use of knowledgebased techniques based on Web ontologies. As formal and web-wide shared conceptualisations, ontologies 
facilitate automated integration and meaningful retrieval of multimedia — both content and metadata — from different sources.

Searching in digital libraries has been widely studied for several years, mostly focusing on retrieving textual information using text-based methods. These queries can be complemented and improved with advanced retrieval methods using content based descriptors extracted from the audiovisual information by applying signal processing, even though some knowledge management and representation of the content is necessary. Moreover, from the service and content providers' point of view, multimedia metadata represents an addedvalue to audiovisual assets, but then again manual annotation is a labour-intensive and error-prone task. Thus, managing audiovisual essence implies to structure its associated metadata using description schemes, taxonomies and ontologies, in order to organize a meaningful data knowledge representation.

In addition to the syntactic interoperation, which is achieved through the standards, semantic interoperation, which is achieved through the integration of domain knowledge expressed in the form of domain ontologies, is also needed for providing efficient retrieval and filtering services. The domain knowledge is subsequently utilized for supporting semantic personalization, retrieval and filtering and has been shown to enhance the retrieval precision (Tsinaraki, Polydoros and Christodoulakis 2007).

This chapter describes the representation of multimedia content descriptions that are structured according to the MPEG-7 metadata description model and expressed using the Semantic Web languages. The rest of the chapter is structured as follows: Section 2 provides an overview of the MPEG-7. The general purpose approaches for multimedia content description that are supported by the MPEG-7 standard are presented as well as the limitations of the current MPEG-7 version (mainly a lack of explicit semantics). Section 3 presents the existing Web Ontology Languages, while Section 4 outlines the efforts made to move the MPEG-7 standard into the Semantic Web. In our case, this is accomplished by interpreting and expressing the informal MPEG7 semantics using Semantic Web languages. An approach for mapping XML Schema (Fallside 2001) constructs to OWL constructs is presented in Section 5, while. Section 6 presents two use cases that show the benefits of this approach, including semantic integration and retrieval in the music domain. An integrated ontological infrastructure for the semantic description of multimedia content is presented in section 7. This infrastructure allows for combining the general-purpose MPEG-7 constructs with domain and application specific knowledge through the systematic representation of this knowledge in the form of Web Ontology Language (OWL) (McGuinness and F. van Harmelen 2004) domain and application ontologies integrated with the MPEG-7 semantics. The chapter conclusions are presented in section 8.

\section{Multimedia Content Description using MPEG-7}

MPEG-7, formally named Multimedia Content Description Interface, is an ISO/IEC standard developed by the Moving Picture Experts Group (MPEG), the committee that also developed the audiovisual standards: MPEG-1, MPEG-2, MPEG-4 and MPEG-21. MPEG-7 aims to create a standard for the description of the multimedia content. The main goal of the MPEG-7 standard is to provide structural and semantic description mechanisms for multimedia content (Salembier, Manjunath, and Sikora 2002).

The MPEG-7 standard provides content description for audiovisual content, defining normative elements such as Descriptors, DescriptionSchemes and a Description Definition Language (DDL). The DDL constitutes the basic building blocks for the MPEG-7 meta-data language. Descriptors are designed for describing different types of information; low-level audiovisual features, high-level semantic objects, content management and information about storage media. Description Schemes are used to group several Descriptors (and Description Schemes) into structured semantically units using the DDL. Ideally, most descriptors corresponding to low-level features would be extracted automatically, whereas human intervention would be required for producing high-level descriptors. 
The standard is divided into four main components: the DDL, the Audio part, the Visual part, and the information about how these elements are combined in a multi-media scenario - a set of Multimedia Description Schemes that includes all the descriptors for capturing the semantic aspects of multimedia contents, e.g. places, actors, objects, events, etc. Thus, the creation of MPEG-7 documents allows a user to query and retrieve (parts of) multimedia and audiovisual information.

In the rest of this section, we discuss media object information description in subsection 2.1, text-based media description in subsection 2.2, low-level feature based media description in subsection 2.3, semantic based media description in subsection 2.4, and retrieving MPEG-7 descriptions in subsection 2.4.

\subsection{Media Object Information Description}

Of special interest is the part 5 of the standard, named MultimediaDescriptionSchemes (MPEG-7 MDS, ISO/IEC 2003). This part includes a set of description tools dealing with generic features and multimedia descriptors. Fig. 1 depicts all the components of the MDS. The Basic elements component includes basic data types, such as media localization, time format, free text annotations, etc. It includes, also, the classification schemes (CS) descriptors. CS descriptors define a scheme for classifying a subject area with a set of terms, organized into a taxonomy. Similar to the WordNet linguistic ontology, basic relationship among the taxonomy terms are available (e.g. narrow and broader terms, and synonyms).

Among the main components of the MDS are the ContentManagement and Description schemes. The Content Management descriptors allow describing the life cycle of multimedia content, from its creation to its usage. They include Media Information to describe storage format, media quality, media location, etc. Moreover, Content Management schemes allow gathering editorial data about the creation and production process of the content. Content Description schemes describe the structural aspects (spatial, temporal and media source structure of the multimedia content) and the semantic aspects.

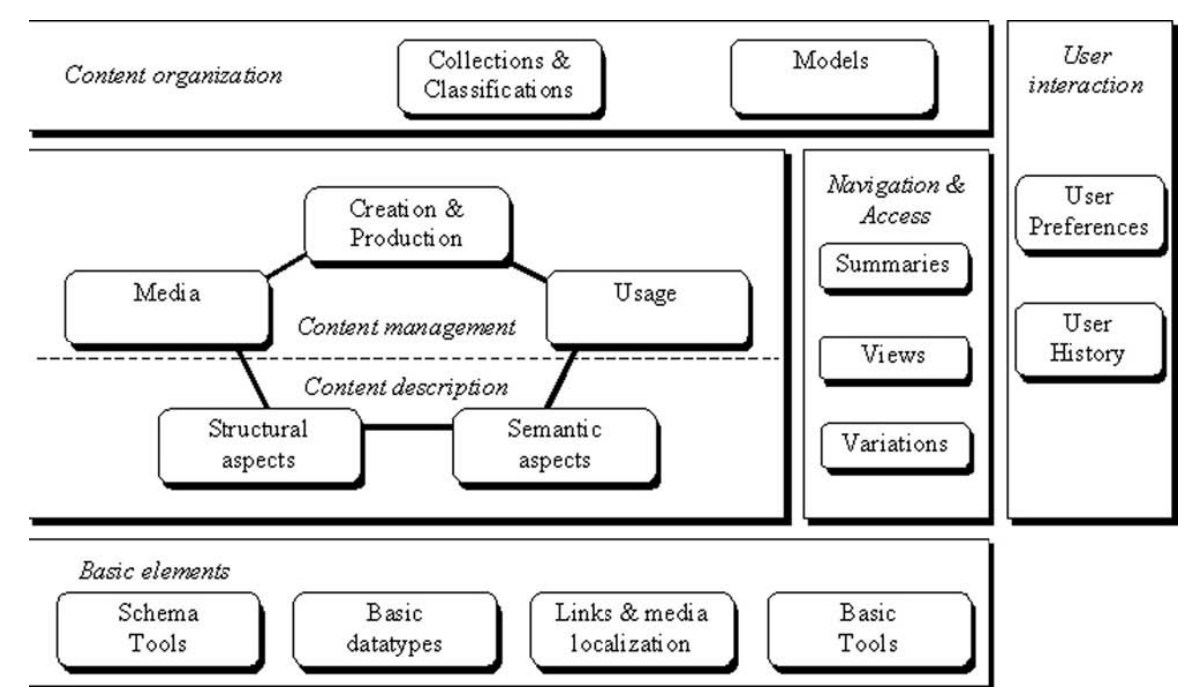

Fig. 1. Main elements of the MPEG-7 Multimedia Description Schemes

In detail, the media object information consists of: 
- The Media Information, which is captured in one of the MediaInformation, MediaInformationRef and MediaLocator elements. The media information consists of the media identification, which allows to uniquely identifying the media object and its locator, and the media profile, which provides media-related information (including media format, quality etc.).

- The Creation Information, which is captured in one of the CreationInformation and CreationInformationRef elements. The creation information consists of information about the media object creation (including title, creators, abstract etc.), classification (including genre, subject, language etc.) as well as information about related material.

- The Structural Information, which is captured in the StructuralUnit element and describes the role of the current multimedia object (segment) within the information context. Thus, the StructuralUnit may take values like "scene”, "shot”, "story” etc.

- The Usage Information, which is captured in one of the UsageInformation and UsageInformationRef elements. The usage information consists of information about the rights associated with the multimedia object, its financial results, its availability and its usage record.

- Information regarding the importance of the multimedia content from specific points of view. This information is captured in the PointOfView element.

- The Relationships of the multimedia content with other media or metadata items as well as the relationships of the semantic entities describing the multimedia content. This information is captured in the Relation element, which associates the media object descriptions with instances of the RelationType that represent relationships. A relationship may be directed or undirected and features a relationship type, the target and the source of the relationship and the strength of the relationship. The standardized MPEG-7 relationship types are more than 100 and are classified into: (a) Basic relationship types (equals, inside, refines etc.), which are specified in the BaseRelation CS; (b) Graph node relationship types (identity, equivalent etc.), which are specified in the GraphRelation CS; (c) Spatial relationship types (over, below, north etc.), which are specified in the SpatialRelation CS; (d) Temporal relationship types (precedes, overlaps, contains etc.), which are specified in the TemporalRelation CS; and (e) Semantic relationship types (shows, agent, causer etc.), which are specified in the SemanticRelation CS.

- The Matching Hints that allow expressing the criteria for matching the multimedia content with low-level audio and visual descriptors. This information is captured in the MatchingHint element.

As an example, consider the MPEG-7 image description of Fig. 2, where Chrisa is shown to write an article. The image description consists of the MediaLocator element, where the image location (http://www.music.tuc.gr/img01.jpg) is specified, and the CreationInformation element, where the image title (Image showing Chrisa writing an article) is specified in its Title element.

\subsection{Text-based Media Description}

In this subsection we describe the text-based multimedia content description capabilities that are provided by the MPEG-7 MDS (ISO/IEC 2003). The textual annotations are represented by the TextAnnotation element of the MPEG-7 segment descriptions. An MPEG-7 textual annotation consists of the following elements, each of which may occur arbitrary times:

- The FreeTextAnnotation element, which represents free text annotations.

- The StructuredAnnotation element, which represents structured textual annotations in terms of who (people and animals), what object, what action, where (places), when (time), why (purpose) and how.

- The KeywordAnnotation element, which represents keyword annotations.

- The DependencyStructure element, which represents textual annotations with a syntactic parse based on dependency structures. 
The confidence in the correctness of a textual annotation and its relevance to the multimedia object being described are represented, in the $[0,1]$ range, by the confidence and relevance attributes of the textual annotation.

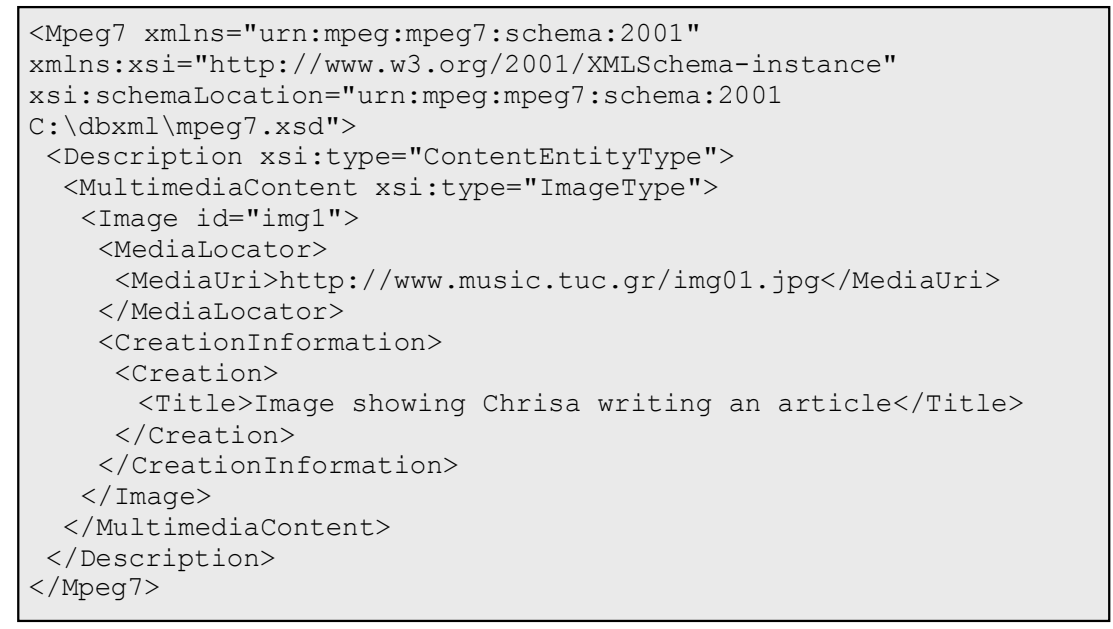

Fig. 2. MPEG-7 Image Description Example

As an example, consider the textual part of the MPEG-7 image description of Fig. 2, which is shown in Fig. 3. The textual annotation consists of a free text annotation (captured in the FreeTextAnnotation element) and a structured annotation (captured in the StructuredAnnotationelement).

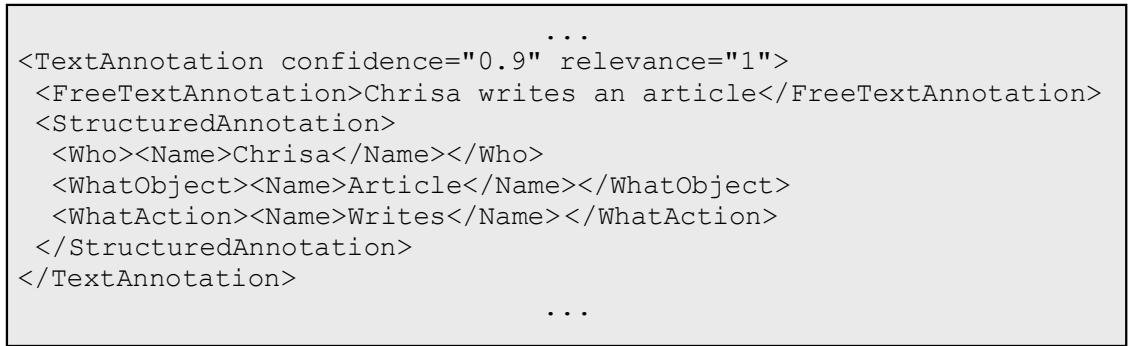

Fig. 3. Textual Part of the MPEG-7 Image Description of Fig. 2

\subsection{Low-level Feature based Media Description}

The MPEG-7 (ISO MPEG Group) allows associating, in the MPEG-7 multimedia object descriptions, lowlevel visual and audio features with the media objects being described. According to the MPEG-7 MDS (ISO/IEC 2003), the MPEG-7 descriptions that describe (segments of) multimedia objects having a visual component (e.g. images, videos, audiovisual segments etc.) may represent the visual features of the described (segments of) multimedia objects through the VisualDescriptor and the VisualDescriptionScheme elements using, respectively, visual descriptors and visual description schemes. The MPEG-7 descriptions that describe (segments of) multimedia objects having an audio component (e.g. audio segments, audiovisual segments etc.) may represent the audio features of the described (segments of) multimedia objects through the 
AudioDescriptor and the AudioDescriptionScheme elements using, respectively, audio descriptors and audio description schemes. In the rest of this subsection we will present the low-level feature based multimedia content description capabilities that are provided by the MPEG-7, focusing on the visual features.

A set of basic low-level descriptors are defined in the MPEG-7 Visual, including the basic Color Descriptors, the basic Texture Descriptors, the basic Shape Descriptors and the basic Motion Descriptors.

MPEG-7 also provides supplementary textual structures for colour spaces, colour quantization and multiple $2 \mathrm{D}$ views of $3 \mathrm{D}$ objects. It also allows for using static (image) descriptors on video content and for the spatial as well as the temporal localization of media object descriptors.

\subsection{Semantic-based Media Description}

In this subsection we describe the semantic-based multimedia content description capabilities provided by the MPEG-7 MDS (ISO/IEC 2003). The semantic multimedia content descriptions are represented by the Semantic element of the MPEG-7 segments, where a set of semantic entities describing the segment content may be defined or referenced. It has been shown in (Tsinaraki, Polydoros, Kazasis and Christodoulakis 2005) that the MPEG-7 semantic description capabilities allow, in addition to the representation of semantic multimedia content descriptions, the representation of domain ontologies using pure MPEG-7 constructs (details in this methodology are provided in section 7).

The semantic entities participating in MPEG-7 descriptions are instances of the subtypes of the abstract type SemanticBaseType, which represent semantic entities of specific types in a narrative world. The $A b$ stractionLevel element of the SemanticBaseType specifies if a semantic entity is abstract or concrete. AbstractionLevel has one attribute, Dimension, of non-negative integer type. When AbstractionLevel is not present in a semantic description, the description refers to specific audiovisual material. When AbstractionLevel.Dimension=0, it is a description of a reusable semantic entity (e.g. the person Chrisa) that is referenced from every segment where the entity appears. When AbstractionLevel has a non-zero Dimension, it specifies classes for the description of abstract semantic entities (e.g. the Article semantic entity, with $A b$ stractionLevel.Dimension=1, represents the class of the articles). The subtypes of SemanticBaseType that represent different types of semantic entities are:

- The SemanticType, which is a concrete type used for the description of collections of semantic entities.

- The AgentObjectType, which is a concrete type used for the description of the actors that appear in a segment. The actors are specified in the Agent element of AgentObjectType. Actors in general are represented using the subtypes of the abstract type AgentType. PersonType, OrganizationType and PersonGroupType are the subtypes of AgentType and are used for the representation of persons (e.g. a student), organizations (e.g. a university) and groups of persons.

- The ObjectType, which is a concrete type used for the description of objects and object abstractions in the material world (e.g. a desk).

- The EventType, which is a concrete type used for the description of events that take place in a semantic world (e.g. writing).

- The ConceptType, which is a concrete type used for the description of concepts present in an audiovisual segment (e.g. co-operation).

- The SemanticStateType, which is a concrete type used for the description of a state of the world described in an audiovisual segment and the parametric description of its features (e.g. the average of a student's grades before and after an examination period).

- The SemanticPlaceType, which is a concrete type used for the description of a place in a semantic world (e.g. Crete).

- The SemanticTimeType, which is a concrete type used for the description of semantic time (e.g. New Year's Eve). 
As an example, consider the semantic part of the MPEG-7 image description of Fig. 2, which is shown in Fig. 4.

Notice that the ChrisaArticle object is the result of the Writes event. The agent of the event is the person represented by the Chrisa semantic entity.

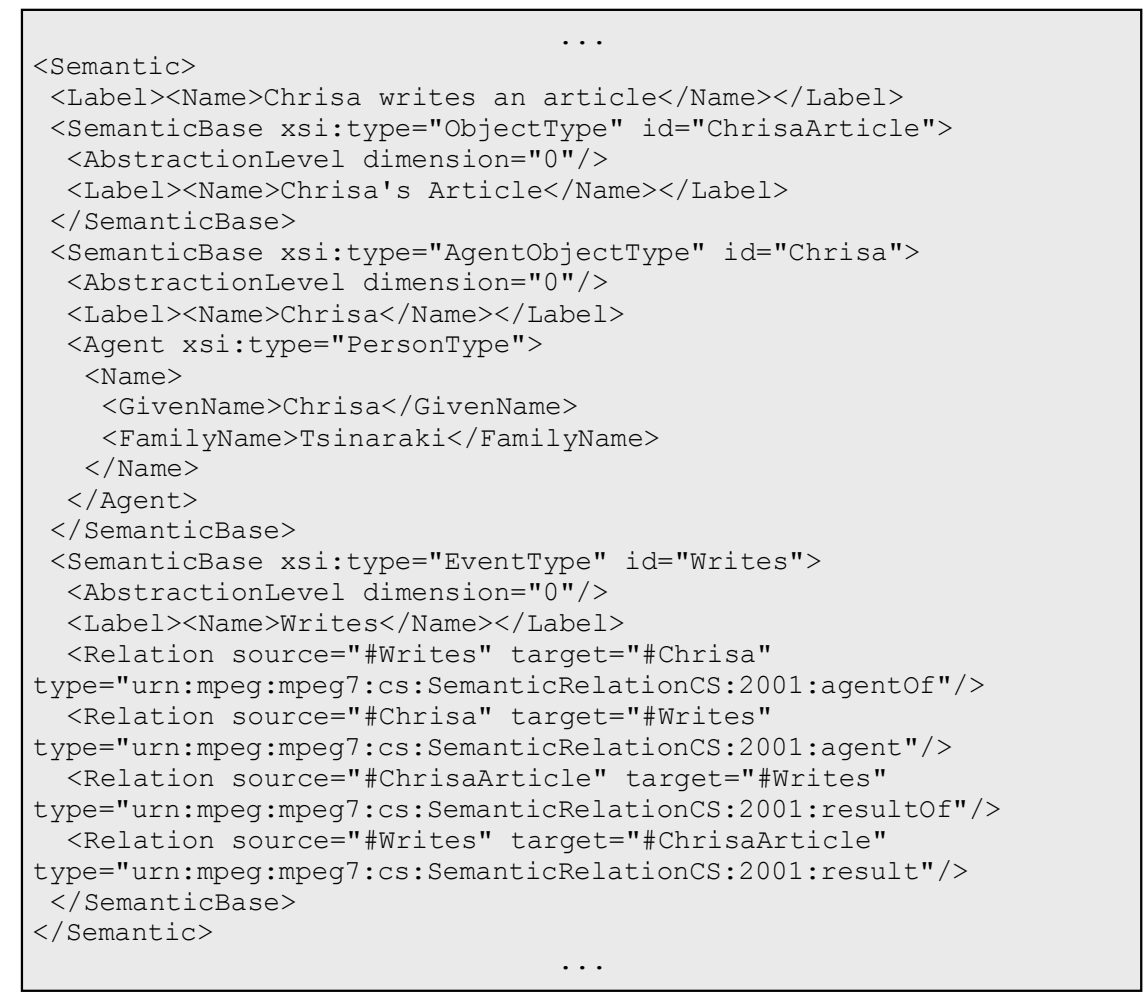

Fig. 4. Semantic Part of the MPEG-7 Image Description of Fig. 2

Semantic entity (abstract or concrete) definitions may occur either in the context of segment descriptions or in independent semantic descriptions. The semantic entity definitions occurring in independent semantic descriptions may then be referenced from the segment descriptions they appear in. This is very useful both for the ontology classes and for the reusable semantic entities.

\subsection{Retrieving information from MPEG-7 descriptions}

The eXtensive Markup Language (XML) has been adopted as the format to represent MPEG-7 descriptors. Also, MPEG-7 DDL is an extension of the W3C XML Schema. XML Schema provides the means for defining the structure of XML documents, that is; simple and complex data types, type derivation and inheritance, element occurrence constraints and, finally, namespace-aware for elements and attributes declarations. MPEG-7 DDL extends the XML Schema and covers the ability to define array and matrix datatypes, and provides specific temporal descriptions (by means of the basicTimePoint and basicDuration types).

The set of MPEG-7 XML Schemas defines 1182 elements, 417 attributes and 377 complex types. The size of this standard makes it quite difficult to manage. Moreover, the use of XML technologies implies that a 
great part of the semantics remains implicit. Therefore, each time an MPEG-7 application is developed, semantics must be extracted from the standard and re-implemented.

Next two examples depict how to retrieve information from MPEG-7 MDS documents using the XQuery (Simeon et. al. 2007) language and an XML database. First example 1 shows an expression to retrieve MPEG-7 audiovisual segments containing any media information. The output is presented as simple HTML code, containing a link to the media file - with the title and type of file as the text link.

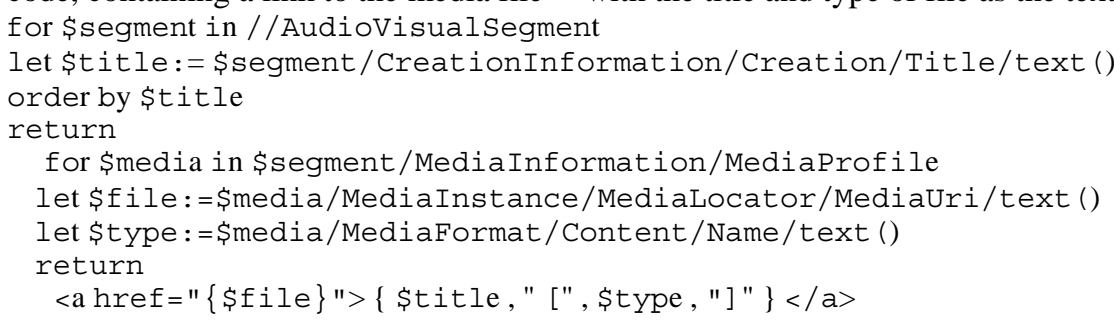

Listing 1. XQuery expression to retrieving a list of multimedia items (title and format type).

The second example shows an XQuery expression to retrieve all MPEG-7 person agents, whose role is Singer, and the characters they play. This query uses a taxonomy that defines different type of singers' role (soprano, contralto, tenor and bass):

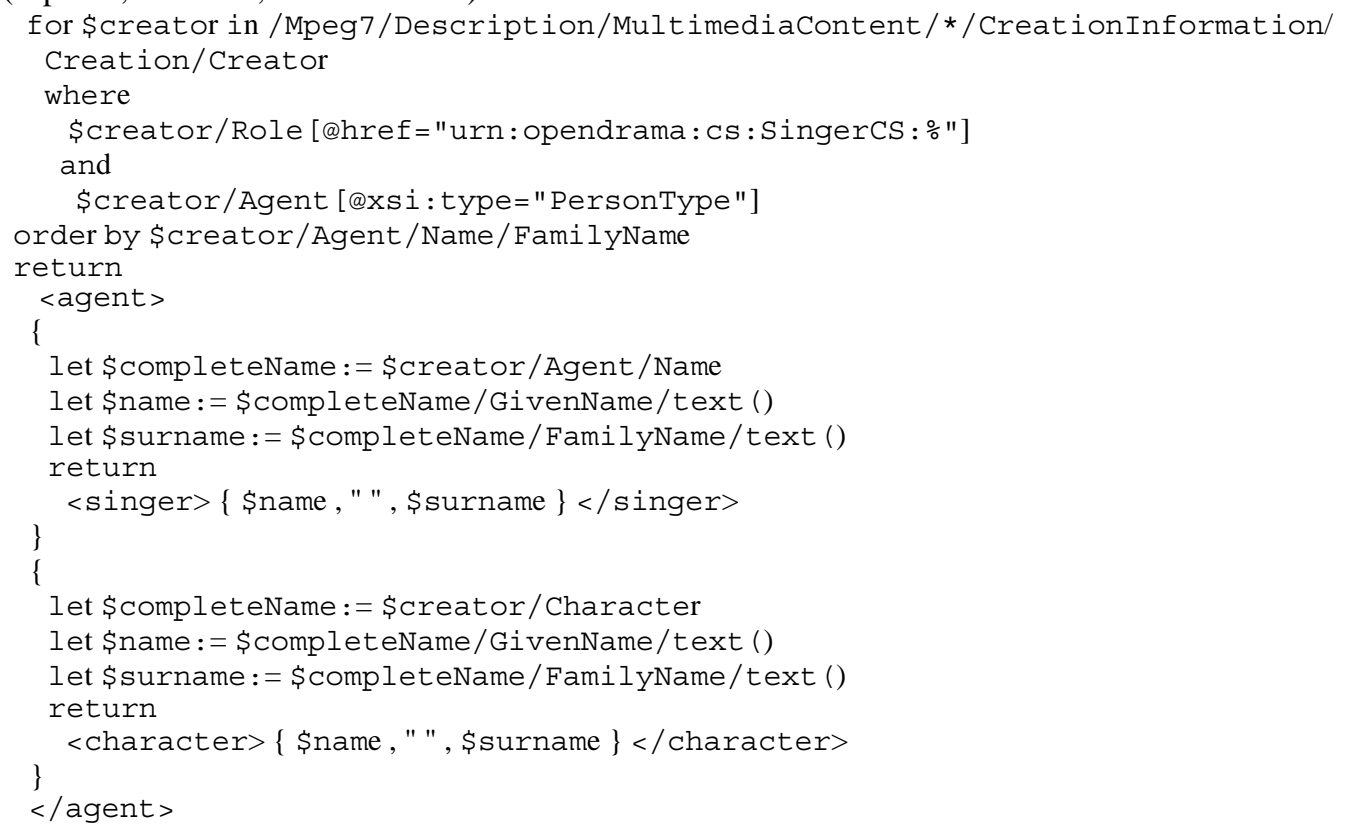

Listing 2. XQuery example to retrieving the singers and the characters they play.

Previous examples only illustrate one kind of difficulty derived from the use of just syntax-aware tools. In order to retrieve any kind of MPEG-7 SegmentType descriptions from an XML database, one must be aware of the hierarchy of segment types and implement an XQuery that covers any kind of multimedia seg- 
ment (i.e. Audio-VisualType VideoSegmentType, AudioSegmentType, etc.). On the other hand, once the hierarchy of segments is explicitly defined in an ontology (e.g in OWL form), semantic queries benefit from the, now, explicit semantics. Therefore, a semantic query for SegmentType will retrieve all the subclasses without requiring additional efforts. This is necessary because, although XML Schemas capture some semantics of the domain they model, XML tools are based on syntax. The captured semantics remain implicit from the XML processing tools point of view. Therefore, when an XQuerysearches for a SegmentType, the XQuery processor has no way to know that there are many other kinds of segment types that can appear in its place, i.e. they are more concrete kinds of segments. At this stage, a possible solution to avoid this is to use wildcards' syntax (see second and fifth lines of the example 2.2). However, this corresponds to a unconstrained generalization, i.e. any element satisfies it and it is not possible to constraint it to just a kind of elements, e.g. all the AudioVisualType subtypes.

Therefore, MPEG-7 constitutes a valuable starting point for more specific developments as it can be seen as an "upper-ontology" for multimedia. However, the lack of explicit semantics makes MPEG-7 very difficult for third party entities to extend in an independent way. This lack of facilities for easy extension has been one of the main motivations to build solutions that make MPEG-7 semantics formal and thus easily machineprocessable. Some solutions to this problem are detailed in section 4.

\section{Web Ontology Languages}

The World Wide Web has changed the way people communicate with each other. Most of todays' Web content is suitable for human consumption. Keyword-based engines have helped users to find the information they are seeking on the net. Yet, search engines present some limitations: the results are single web pages, results are highly sensitive to the vocabulary (semantically similar queries should return similar results), and usually there is a high recall and low precision of the result set (i.e. there is too much noise on the webpage results) (Antoniou, G. and Harmelen, F. van 2004).

The main problem of the current Web, at this stage, is that the meaning of the content is not accessible by machines. Information retrieval and text processing tools are widely used, but there is still difficulties when interpreting sentences, or extracting useful information for users. The development of the Semantic Web, with machine- readable content, has the potential to revolutionize the current World Wide Web and its use.

\subsection{Overview of the Semantic Web}

The definition and vision that had Tim Berners Lee (Berners-Lee 1999) is that the Semantic Web is an extension of the current web in which information is given well-defined meaning, better enabling computers and people to work in cooperation. The Semantic Web is a vision: the idea of having data on the Web defined and linked in a way that it can be used by machines not just for display purposes, but for automation, integration and reuse of data across various applications (Berners-Lee, Hendler and Lassila, O. 2001; Shadbolt, BernersLee, and Hall 2006).

The previous ideas and principles to enhance the Web are being put into practice under the guidance of the World Wide Web Consortium (W3C). The next statement presents their view:

"The semantic web is an extension of the current web in which information is given well-defined meaning, better enabling computers and people to work in cooperation. The mix of content on the web has been shifting from exclusively human-oriented content to more and more data content. The semantic web brings to the web the idea of having data defined and linked in a way that it can be used for more effective discovery, automation, integration, and reuse across various applications.Forthe webto reachitsfullpotential, it must evolve into a semantic web, providing a universally accessible platform that allowsdata tobe shared andprocessedby automated tools as well asby peo- 


\section{ple." — W3C Semantic Web Activity Statement}

The Semantic Web technologies have been arranged into a layered architecture. The key technologies include explicit metadata, ontologies, logic and inferencing, and intelligent agents. Each layer, from the bottom to the top, has an increasing level of complexity, yet it offers more expressivity.

The two base layers (Unicode and URI, and the XML family) are inherited from the current Web. Section 2 already has presented some technologies related with XML. The upper layers compose the Semantic Web, over the existing basic technologies. The next sections overview these layers, that is: the Resource Description Framework (RDF), the RDF Schema (RDFS), and the Ontology Vocabulary (OWL).

\subsection{Resource Description Framework}

RDF (Brickley and Guha 2004) vocabulary is similar to other knowledge representation formalisms like conceptual graphs (CG) or semantic nets. CG express meaning in a form that is logically precise, humanly readable, and computationally tractable. CG serve as an intermediate language for translating computer-oriented formalisms to and from natural languages. With a clear graphic representation, they serve as a readable —but formal - design and specification language. The next figure, Fig. 5, shows an example of a semantic net, which relates music bands, artists and basic data:

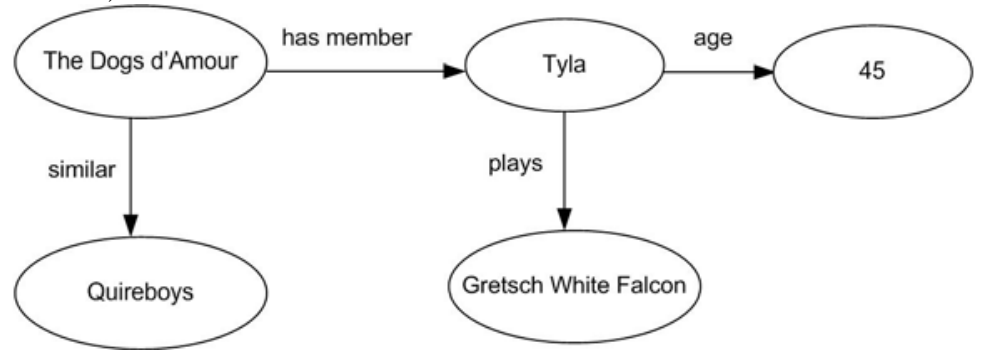

Fig. 5. A semantic net.

Graph representation is a powerful tool for human understanding. However, in our context we need machine-processable representations.

RDF vocabulary allows formally describing the previous example, and even serializing it using the XML language. RDF is, then, a data model for objects (resources) and the relations (properties) between them, and it provides simple semantics. A resource is an object, a thing we want to talk about. A resource has an URI (Universal Resoure Identifier). Properties are a special kind of resource that describe relations between resources (e.g. related with, age, plays, etc.). Properties are identified by URIs.

Statements assert the properties of resources. From a natural language point of view, a statement is composed by a Subject-Predicate-Object triple. From a more computer science point of view, this is equivalent to an Object-Attribute-Value triple, or in this context a Resource-Property-Value triple. A triple [x, $\mathrm{P}, \mathrm{y}]$ is equal to a logical formula $\mathrm{P}(\mathrm{x}, \mathrm{y})$, where the binary predicate $\mathrm{P}$ relates the object $\mathrm{x}$ to the object $\mathrm{y}$. Values can be either resources or literals (e.g. strings).

A possible statement could be: "Oscar Celma is the owner of the web page http://foafing-themusic.iua.upf.edu". This triple is equal to the graph statement:

It is a directed graph, where the nodes correspond to the objects and the labeled arc is a property. The same statement can be represented in XML syntax (also known as RDF/XML):

$<$ rdf:Description

rdf : about="http: //foafing-the-music.iua.upf.edu" > 
$<$ mydomain: owner>Oscar Celma</mydomain: owner >

$</$ rdf :Description $>$

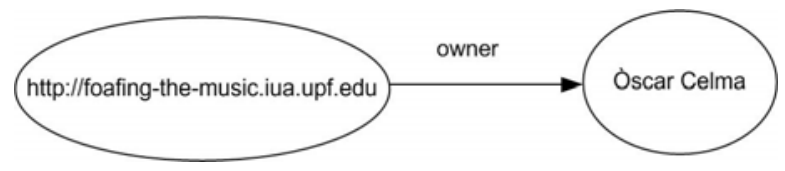

Fig. 4. Graph representation of a triple.

The rdf:Description makes a statement about the resource (a web page) http://foafingthe-music.iua.upf.edu. The property (owner) is used as a tag within the description, and the value is the content of the tag . Moreover, we can describe the person "Oscar Celma" by the resource with URL http://www.mydomain.org/people/\#44521:

$<$ rdf: Description

rdf : about ="http: / / www.mydomain.org/people/\#44521" >

$<$ mydomain: name>Oscar Celma</mydomain:name>

$<$ mydomain:title>Associate

Professor</mydomain:title $>$

$</$ rdf : Description $>$

In this case, the rdf:Description corresponds to two statements about the resource http: / / www . mydomain.org/people/\#44521 (the name, and the title of that person). Now, we can define a course that is taughtby that resource:

$<$ rdf: Description

rdf : about="http: //www.tecn.upf.es/ ocelma/edi2" >

<uni : courseName>Introduction to Databases</uni : courseName>

<uni : creditsNumber $>6<$ /uni : creditsNumber $>$

<uni : isTaughtBy rdf : resource="http://www. mydomain.org/people /\#44521"/>

$</$ rdf :Description $>$

The resulting graph of the three previous examples is depicted in Fig. 5.

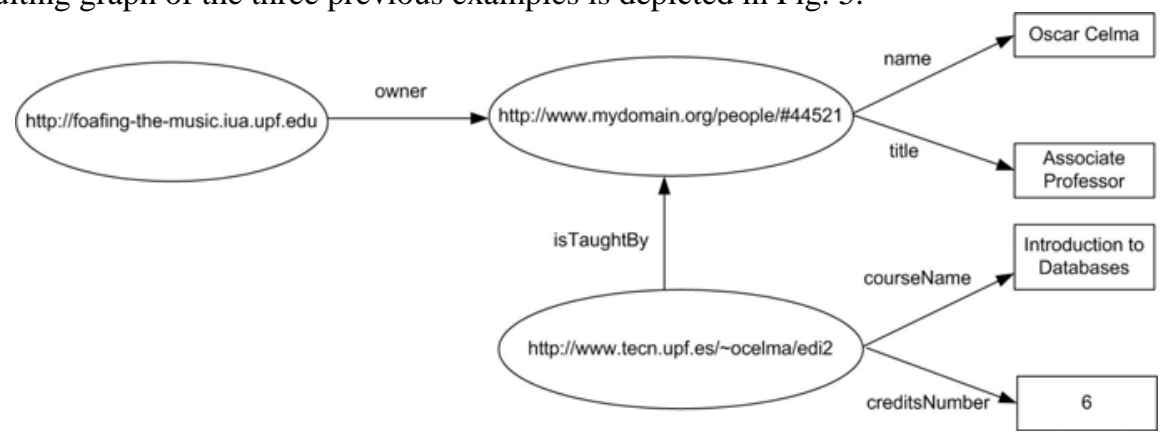

Fig. 5. Graph representation of the previous RDF statements.

By now, we have defined a set of statements, but there are still no restrictions about them. For instance, we should state that the property isTaughtBy is only applied to courses (the subject) and professors (the ob- 
ject), or that an Associate Professor is a particular type of Professor, with some restrictions (maximum number of hours, needs to hold a PhD., etc). The RDF Schema vocabulary is intended to describe this information.

\subsection{RDF Schema}

RDF Schema (RDFS) (Manola and Milles 2004) is a vocabulary for describing properties and classes of RDF resources, and provides hierarchies of such properties and classes. RDFS vocabulary allows defining the semantics of the RDF statements.

As it is common in other disciplines, to describe a particular domain one can use classes and properties. RDFS provides mechanisms to define a particular domain using classes (and properties), hierarchies and inheritance. Classes model the entities (and their restrictions) of the domain, whereas properties provide relationships among the classes. Properties have a domain and range (similarly to mathematical functions), to impose restrictions on the values of the property. Yet, there are some important missing features of RDFS:

- There are no local scope properties: rdf:range defines the range of a property for all classes. We cannot declare range restrictions that apply to some classes only

- There is no disjointness of classes

- Missing boolean combinations of classes: Union, Intersection, and complement

- No cardinality restrictions: restrictions on how many distinct values a property may or must take ("a person has two parents")

- No special characteristics of properties: transitive (greater than), unique (is mother of) and inverse (eats and is eaten by)

These limitations are solved in the OWL language, presented in the next section. To conclude this section, a simile can be established among the existing technologies on the current Web, and the ones proposed by the Semantic Web community: while XHTML language makes the Web behave like a global book when viewed at the worldwide level, RDF and RDF Schema make it behave like a global database. Regarding the data structures, the basic RDF primitive is a directed graph, whereas XML representation is based on a tree. Thus, an RDF graph is on its own basically unrestricted and more powerful, in terms of expressiveness.

\subsection{Ontology Vocabulary}

An ontology is an explicit and formal specification of a conceptualization (Gruber 1993). In general, an ontology describes formally a domain of discourse. The requirements for Ontology languages are: a welldefined syntax, a formal semantics, and a reasoning support that checks the consistency of the ontology, checks for unintended relationships between classes, and automatically classifies instances in classes.

The Web Ontology Language (OWL) has a richer vocabulary description language for describing properties and classes than RDFS. OWL has relations between classes, cardinality, equality, characteristics of properties and enumerated classes. The OWL language is built on RDF and RDFS, and uses RDF/XML syntax. OWL documents are, then, RDF documents.

The next example shows the definition of two classes: <owl : Class rdf : ID="Singer">

<rdfs: subclassof rdf : resource="\#Artist" />

$<$ owl : Class $>$

<owl: Class rdf : ID="Song" />

Object property elements relate objects to other objects. For instance "a singer sings songs". <owl : ObjectProperty rdf : ID="sings"> 


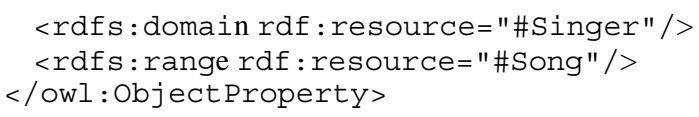

Data type properties relate objects to datatype values. For example, the dataproperty that denotes the age of an Artist:

<owl : DataProperty rdf : ID="age">

$<$ rdfs: domain rdf : resource="\#Artist" $/>$

$<$ rdfs : range rdf : resource=" \&xsd; nonNegativeInteger" />

<owl : DataProperty>

Property restrictions on classes are based on the use of rdfs:subclassOf. To say that class $\mathrm{C}$ satisfies certain conditions is equivalent to state that $C$ is a subclass of C', where C' collects all objects that satisfy the conditions. For instance, a restriction on the kind of values the property can take:

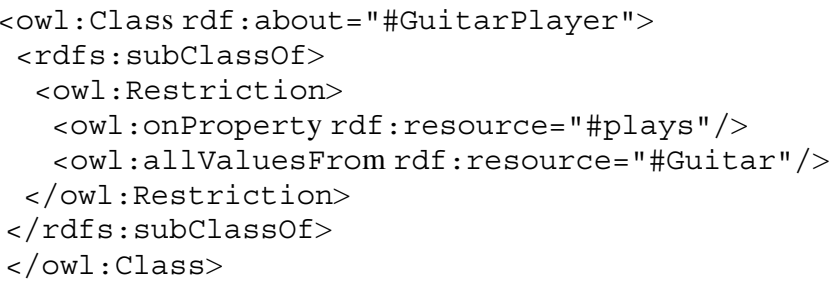

Or cardinality restrictions (a music band is composed by, at least, two members): <owl : Class rdf : about = "\#Band">

OWL offers some special properties, such as: owl:TransitiveProperty (e.g. "has better grade than”, "is taller than", "is ancestor of", etc.), owl:SymmetricProperty (e.g. "has same grade as", "is sibling of", etc.), owl:FunctionalProperty (a property that has almost one value for each object, e.g. "age"), and owl:InverseFunctionalProperty (a property for which two different objects cannot have the same value, e.g. "socialSecurityNumber"). For example, a playedwith property is symmetric:

<owl : Object Property raf : ID="playedWith">

<rdf : type rdf : resource=" \&owl; SymmetricProperty" />

<rdfs: domain rdf : resource="\#Artist" />

<rdfs:range rdf:resource="\#Artist" / >

$</$ owl : ObjectProperty $>$

There are three different OWL sublanguages. Each sublanguage offers a level of expressivity. OWL Full is the most expressive of the three sublanguages. There are no special constraints about how the OWL primitives can be used. Therefore, the greatest level of expressivity of the language can be achieved. However, on the other hand the language becomes undecidable, so efficient reasoning is not guaranteed. 
OWL DL is based on Description Logics. It has vocabulary partitioning, that is: any resource is allowed to be only a class, a data type, a data type property, an object property, an individual, a data value, or part of the built-in vocabulary. And, there is explicit typing in OWL DL, so the vocabulary partitioning must be stated explicitly.

Property separation implies that the following can never be specified for data type properties: owl:inverseOf, owl:FunctionalProperty, owl:InverseFunctionalProperty, and owl:SimmetricProperty. And there is a restriction for anonymous classes: they are only allowed to occur as the domain and range of either owl:equivalentClass, owl:disjointWith and as the range of $r d f$ s:subClassOf.

These constraints on how OWL primitives are combined guarantee that to reason on OWL DL expressions is decidable and tractable, i.e. it will terminate in a finite and not too big amount of time. This is so because OWL DL is in the family of Description Logics. Description Logics allow specifying a terminological hierarchy using a restricted set of first order formulas. Restrictions make that Description Logics have nice computational properties but the inference services are restricted to subsumption and classification. Subsumption means, given formulae describing classes, the classifier associated with certain description logic will place them inside a hierarchy. On the other hand, classification means that given an instance description, the classifier will determine the most specific classes to which the particular instance belongs.

Finally, OWL Lite has the same restrictions as OWL DL plus it is not allowed to use owl:oneOf, owl:disjointWith, owl:UnionOf, owl:complementOf nor owl:hasValue, Regarding cardinality statements: only values 0 and 1 are possible. These additional constraints reduce even more the expressivity of the language but, on the other hand, make reasoning more efficient.

\section{MPEG-7 Ontologies}

As it has been shown in Section 2, MPEG-7 allows for the semantic annotation of multimedia content and the systematic representation of domain knowledge using MPEG-7 constructs. The domain knowledge is usually expressed today in the form of domain ontologies, and several ontology description languages have been proposed, based on the OWL language presented in Section 3. Thus, it is expected that both, many OWL domain ontologies will be developed, as well as that many developers will be familiar with OWL and will use it for ontology definition. It is therefore very important for the multimedia community to have a methodology for the interoperability of OWL with MPEG-7 and for the integration of domain knowledge expressed in OWL within MPEG-7. This way, the MPEG-7 constructs will become Semantic Web objects and the Semantic Web tools (such as reasoners) and methodologies may be used with MPEG-7. This feature is useful for several applications, e.g. knowledge acquisition from multimedia content. In this section we present the ontologies expressed in the Semantic Web languages that capture (fully or partially) the MPEG-7 semantics. As a consequence, although the MPEG-7 is a standard, hence it enhances interoperability at least at the syntactic level, the several different ontological MPEG-7 representations are not standard, and are not compatible or interoperable with each other. Thus, a new interoperability issue appears, which is discussed in detail in the Harmonization of Multimedia Ontologies activity of the AceMedia project (AceMedia 2007) and (Celma et al 2007).

Chronologically, the first efforts towards a semantic formalisation of MPEG-7 were carried out, during the MPEG-7 standardisation process, by Jane Hunter (Hunter 1999). The proposal used RDF (Brickley and Guha 2004) and RDF Schema (Manola and Milles 2004) to formalise a small part of MPEG-7, and later incorporated some DAML+OIL constructs (McGuinness, Fikes, Hendler and Stein 2002) to further detail their semantics (Hunter 2001), where the DAML+OIL ontology definition language was used to partially describe the MPEG-7 MDS and Visual metadata structures. The ontology has been recently translated in OWL. However, it continues to show one of its major shortcomings, the limited coverage of the MPEG-7 constructs. 
Another proposal based on RDF/RDFS that captures the MPEG-7 Visual has been presented in (Simou, Tzouvaras, Avrithis, Stamou and Kollias 2005). The same shortcomings are observed due to the expressivity limitations of RDF/RDFS. Consequently, this ontology also provides limited support for the representation of MPEG-7 formal semantics.

An OWL Full ontology that captures the whole MPEG-7 standard was presented in (García and Celma 2005). This ontology has been automatically produced using the mappings from XML Schema constructs to the OWL constructs, which are detailed in Section 5. This mapping is complemented with an XML to RDF one that makes it possible to map existing MPEG-7 data to RDF data based on the previous ontology ${ }^{1}$.

The disadvantage of modelling the whole standard is that this ontology is OWL-Full, which means that computational completeness and decidability of reasoning are not guaranteed. However, this limitation is unavoidable due to the structure of the MPEG-7 standard XML Schemas. The only way to avoid it, if all the semantics implicit in the schemas are formalised, is to restrict the ontology to just a part of the standard.

This is the approach of the OWL-DL ontology presented in (Tsinaraki, Polydoros and Christodoulakis 2004b), which captures the full MPEG-7 MDS (including the classification schemes) and just the parts of the MPEG-7 Visual and Audio that are necessary for the complete representation of the MPEG-7 MDS. The ontology was manually developed, according to a methodology that allows the transformation of the XML Schema constructs of MPEG-7 in OWL-DL.

The methodology consists of the following steps:

- The MPEG-7 simple datatypes are imported from the XML Schema syntax, as OWL does not directly support simple type definition.

- The MPEG-7 complex types are represented as OWL classes, which have the complex type names as identifiers. The attributes and the simple type elements (of type string, integer etc.) of the complex types are represented as OWL datatype properties that have the OWL classes that represent the complex types as domain and the simple types as range. The complex type elements are represented as OWL object properties that have the OWL classes that represent the complex type as domain and the OWL classes that represent the element types as range (if the latter do not already exist, it is defined from scratch).

- For the representation of the subtype/supertype relationships that hold for a complex type, the following actions are performed: (a) If the complex type is a subtype of another complex type, the subclass relationship is represented using the OWL/RDF subclassing construct; and (b) If the complex type is a subtype of a simple type, a datatype property is defined that has as identifier "type_nameContent", where type_name is the type of the supertype (e.g. string, integer etc.). The datatype property has the supertype as range and the OWL class that represents the complex type as domain.

- The XML Schema restrictions are transformed to the analogous OWL constructs; Thus, a fixed attribute value is transformed to an OWL "hasValue" restriction, and the minOccurs/maxOccurs attributes are transformed to either simple cardinality restrictions (i.e. cardinality, minCardinality and maxCardinality) or groups of cardinality restrictions, grouped using the OWL unionOf (in case of choices) and intersectionOf (in case of sequence) constructs.

- The MPEG-7 Classification Schemes are represented as individuals of the ClassificationSchemeType class, which represents the homonym MPEG-7 type that specifies the structure of the classification schemes.

The main advantage of the above methodology is that, thanks to the manual effort, an OWL-DL ontology has been produced, which accurately captures the semantics of the MPEG-7 constructs (including both the named and unnamed - nested - ones).

\footnotetext{
${ }^{1}$ MPEG-7 Ontologies, http://rhizomik.net/ontologies/mpeg7ontos
} 


\section{Mapping Approach}

The approach used to map XML Schema constructs in the MPEG-7 standard to OWL constructs is based on a generic XML Schema to OWL mapping combined with an XML to RDF translation. It has already shown its usefulness with other quite big XML Schemas in the Digital Rights Management domain, such as MPEG21 and ODRL (García, Gil, Delgado 2007), and also in the E-Busines domain (Garcia, Gil 2007).

The main contribution of this approach is that it allows to take profit from the great amount of metadata that has been already produced by the XML community. There are many attempts to move metadata from the XML domain to the Semantic Web. Some of them just model the XML tree using the RDF primitives (Klein 2002). Others, concentrate on modelling the knowledge implicit in XML languages definitions, i.e. DTDs or the XML Schemas, using web ontology languages (Amann et al. 2002; Cruz et al. 2004; Halevy 2003). Finally, there are attempts to encode XML semantics integrating RDF into XML documents (Lakshmanan 2003, Patel-Schneider 2002).

None of the previous approaches facilitates an extensive transfer of XML metadata to the Semantic Web in a general and transparent way. Their main problem is that the implicit interpretation of XML Schema in terms of RDF(S) and OWL semantics is not formalised when XML metadata instantiating this schemas is mapped. Therefore, they do not take profit from the XML semantics and produce RDF metadata almost as semantics-blind as the original XML. Or, on the other hand, they capture these semantics but they use additional ad-hoc semantic constructs that produce less transparent metadata. Therefore, we have chosen the XML Semantic Reuse methodology (García 2006) implemented by the ReDeFer ${ }^{2}$ project. It combines an XML Schema to web ontology mapping, called XSD2OWL, with a transparent mapping from XML to RDF, XML2RDF. The ontologies generated by XSD2OWL are used during the XML to RDF step in order to generate semantic meta-data that makes XML Schema semantics explicit. Both steps are detailed next. To conclude, in order to improve the transfer from MPEG-7 XML metadata to the Semantic Web, there is also a simple MPEG-7 Classification Scheme to OWL mapping called CS2OWL. It maps MPEG-7 classification hierarchies, e.g. TV-Anytime ${ }^{3}$ hierarchy of contents or formats, to an OWL hierarchy of classes.

\subsection{XSD2OWL Mapping}

The XML Schema to OWL mapping is responsible for capturing the schema informal semantics. This semantics are derived from the combination of XML Schema constructs. The mapping is based on translating these constructs to the OWL ones that best capture their meaning. These mappings are detailed in Table 1.

The XSD2OWL mapping is quite transparent and captures the semantics implicit in XML Schema following the interpretations in Table 1. The same names used for XML constructs are used for the OWL ones, although in the new namespace defined for the ontology. XSD and OWL constructs names are identical; this usually produces uppercase-named OWL properties because the corresponding element name is uppercase, although this is not the usual convention in OWL.

Table 1. XSD2OWL translations for the XML Schema constructs and their interpretations in terms of the corresponding OWL constructs

\begin{tabular}{crc}
\hline \hline XML Schema & OWL & Shared semantics \\
\hline element | attribute & rdf:Property & Named relation between
\end{tabular}

\footnotetext{
${ }^{2}$ ReDeFer, http://rhizomik.net/redefer

${ }^{3}$ TVAnytime, http://www.tv-anytime.org
} 


\begin{tabular}{|c|c|c|}
\hline & $\begin{array}{l}\text { owl:DatatypeProperty } \\
\text { owl:ObjectProperty }\end{array}$ & nodes or nodes and values \\
\hline element@substitutionGroup & rdfs:subPropertyOf & $\begin{array}{l}\text { Relation can appear in } \\
\text { place of a more general } \\
\text { one }\end{array}$ \\
\hline element@type & rdfs:range & The relation range kind \\
\hline $\begin{array}{l}\text { complexType|group } \\
\text { |attributeGroup }\end{array}$ & owl:Class & $\begin{array}{l}\text { Relations and contextual } \\
\text { restrictions package }\end{array}$ \\
\hline complexType//element & owl:Restriction & $\begin{array}{l}\text { Contextualised restriction } \\
\text { of a relation }\end{array}$ \\
\hline $\begin{array}{l}\text { extension@base | } \\
\text { restriction@base }\end{array}$ & rdfs:subClassOf & $\begin{array}{l}\text { Package concretises the } \\
\text { base package }\end{array}$ \\
\hline $\begin{array}{l}@ \text { maxOccurs } \\
@ \text { minOccurs } \\
\text { none specified }\end{array}$ & $\begin{array}{l}\text { owl:maxCardinality } \\
\text { owl:minCardinality } \\
\text { owl:cardinality(1) }\end{array}$ & $\begin{array}{l}\text { Restrict the number of } \\
\text { occurrences of a relation. } \\
1 \text { implicit if not specified }\end{array}$ \\
\hline $\begin{array}{l}\text { sequence } \\
\text { choice }\end{array}$ & $\begin{array}{l}\text { owl:intersectionOf } \\
\text { owl:unionOf }\end{array}$ & $\begin{array}{l}\text { Combination of relations } \\
\text { in a context }\end{array}$ \\
\hline
\end{tabular}

Therefore, XSD2OWL produces OWL ontologies that make explicit the semantics of the corresponding XML Schemas. The only caveats are the implicit order conveyed by xsd:sequence and the exclusivity of xsd:choice. For the first problem, owl:intersectionOf does not retain its operands order, there is no clear solution that retains the great level of transparency that has been achieved. The use of RDF Lists might impose order but introduces ad-hoc constructs not present in the original metadata. Moreover, as it has been demonstrated in practise, the element ordering does not contribute much from a semantic point of view. For the second problem, owl:unionOf is an inclusive union, the solution is to use the disjointness OWL construct, owl:disjointWith, between all union operands in order to make it exclusive.

Table 2. XML Schema to OWL mapping example (namespaces omitted for readability)

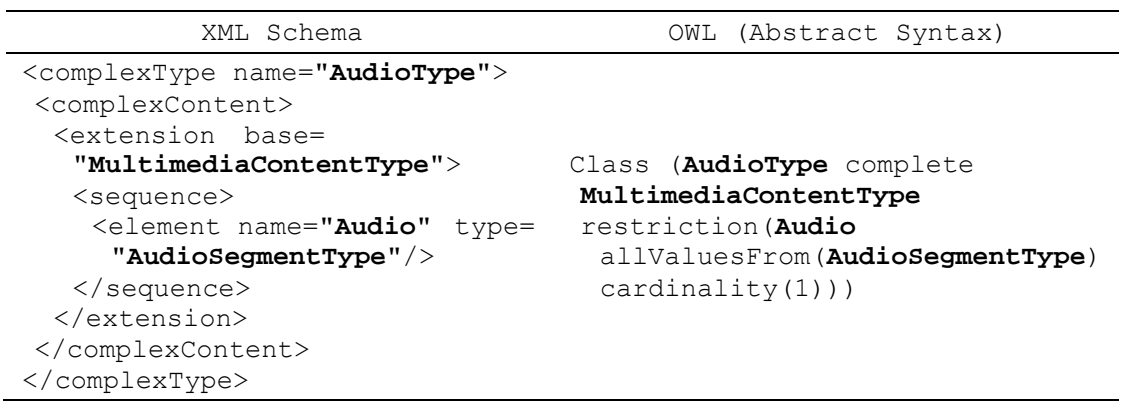

The XSD2OWL mapping has been applied to the MPEG-7 XML Schemas producing the complete MPEG-7 Ontology ${ }^{4}$. This ontology has 2372 classes and 975 properties. The only adjustment that has been done to the automatically generated ontology is to resolve a name collision between an OWL class and a

\footnotetext{
${ }^{4}$ MPEG-7 Ontologies, http://rhizomik.net/ontologies/mpeg7ontos
} 
RDF property. This is due to the fact that XML has independent name domains for complex types and elements while OWL has a unique name domain for all constructs. Moreover, the resulting OWL ontology is OWL-Full because the XSD2OWL translator has been forced to employ rdf:Property for those xsd:elements that have both data type and object type ranges. Table 2 shows and example of an XML Schema ComplexType mapping to the corresponding OWL Class.

\subsection{XML2RDF Mapping}

Once all the XML Schemas for the metadata under consideration are available as mapped OWL ontologies, it is time to map the XML metadata that instantiates them. The intention is to produce RDF metadata as transparently as possible. Therefore, a structure-mapping approach has been selected (Klein 2002). It is also possible to take a model-mapping approach (Tous, García, Rodríguez and Delgado 2005). XML model-mapping is based on representing the XML information set using semantic tools. This approach is better when XML metadata is semantically exploited for concrete purposes. However, when the objective is semantic metadata that can be easily integrated, it is better to take a more transparent approach. Transparency is achieved in structure-mapping models because they only try to represent the XML metadata structure, i.e. a tree, using RDF. The RDF model is based on the graph so it is easy to model a tree using it.

Moreover, we do not need to worry about the semantics loose produced by structure-mapping. We have formalised the underlying semantics into the corresponding ontologies and we will attach them to RDF metadata using the instantiation relation rdf:type.

The structure-mapping is based on translating XML metadata instances to RDF ones that instantiate the corresponding constructs in OWL. The more basic translation is between relation instances, from xsd:elements and xsd:attributes to rdf:Properties. Concretely, owl:ObjectProperties for node to node relations and owl:DatatypeProperties for node to values relations. However, in some cases, it would be necessary to use $r d f$ :Properties for $x$ sd:elements that have both data type and object type values. Values are kept during the translation as simple types and RDF blank nodes are introduced in the RDF model in order to serve as source and destination for properties.

The resulting RDF graph model contains all that we can obtain from the XML tree. It is already semantically enriched thanks to the rdf:type relation that connects each RDF properties to the owl:ObjectProperty or owl:DatatypeProperty it instantiates. It can be enriched further if the blank nodes are related to the owl:Class that defines the package of properties and associated restrictions they contain, i.e. the corresponding xsd:complexType. This semantic decoration of the graph is formalised using rdf:type relations from blank nodes to the corresponding OWL classes.

At this point we have obtained a semantics-enabled representation of the input metadata. The instantiation relations can now be used to apply OWL semantics to metadata. Therefore, the semantics derived from further enrichments of the ontologies, e.g. integration links between different ontologies or semantic rules, are automatically propagated to instance metadata thanks to inference. We will show now how this mapping fits in the architecture for semantic multimedia metadata integration and retrieval.

However, before continuing to the next section, it is important to point out that these mappings have been validated in different ways. First, we have used OWL validators in order to check the resulting ontologies, not just the MPEG-7 Ontology but also many others (García and Gil 2007; García, Gil and Delgado 2007). Second, our MPEG-7 ontology has been compared with hand made ontologies like Jane Hunters' one (Hunter 2001) and Tsinaraki’s (Tsinaraki, Polydoros and Christodoulakis 2004b). This comparison has shown that our mapping captures all the semantics captured by these ontologies and even adds additional details not captured by them in order to get a full formalisation of the semantics in all the MPEG-7 XML Schemas.

Finally, the two mappings have been tested in conjunction. Testing XML instances have been mapped to RDF, guided by the corresponding OWL ontologies from the used XML Schemas, and then back to XML. 
Then, the original and derived XML instances have been compared using their canonical version in order to correct mapping problems.

\section{Use Cases}

Based on the previous XML to Semantic Web mapping, a system architecture that facilitates multimedia metadata integration and retrieval has been built. The architecture is sketched in Fig. 6. The MPEG-7 OWL ontology, generated by XSD2OWL, constitutes the basic ontological framework for semantic multimedia metadata integration and appears at the centre of the architecture. Other ontologies and XML Schemas might be easily incorporated using the XSD2OWL module.

Semantic metadata can be directly fed into the system together with XML metadata, which is translated to semantic metadata using the XML2RDF module. XML MPEG-7 metadata has a great importance because it is commonly used for (automatically extracted) low-level metadata that constitutes the basic input of the system.

This framework has the persistence support of a RDF store, where metadata and ontologies reside. Once all metadata has been put together, the semantic integration can take place, as detailed in Section 6.1. Finally, from this integrated space, higher-level metadata can be inferred and retrieved, as shown in Section 6.2.

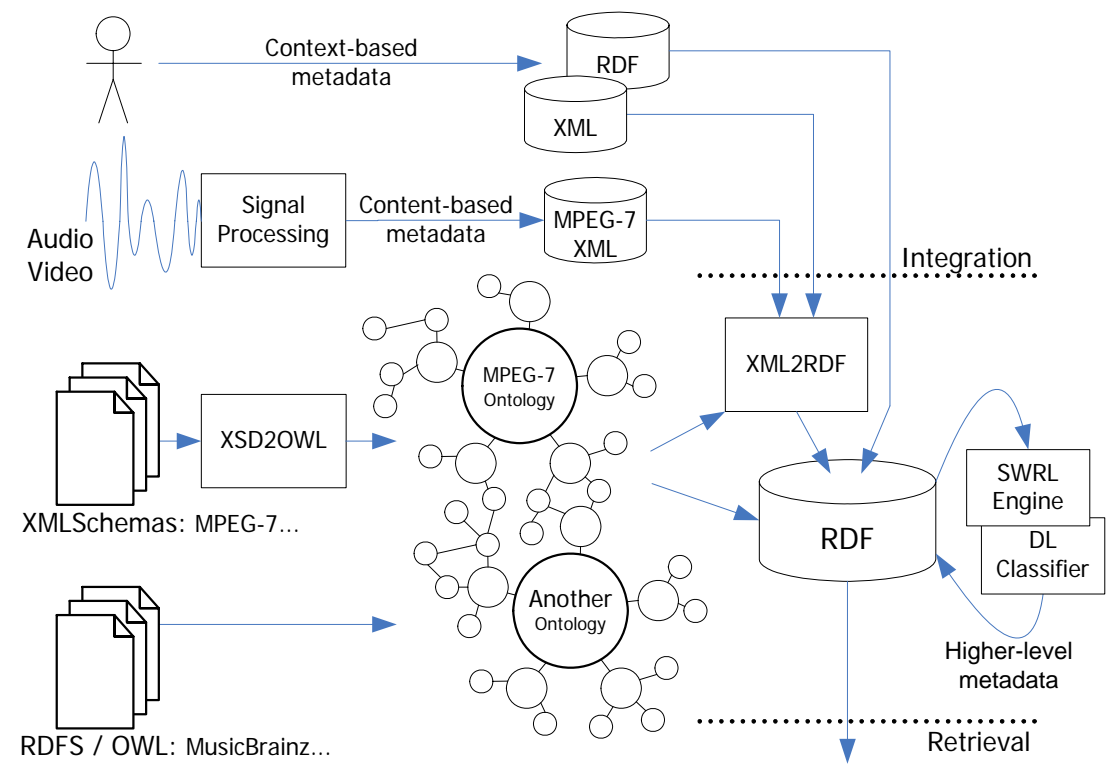

Fig. 6. Metadata integration and retrieval architecture

\subsection{Semantic Integration of Music Metadata}

The problem of integrating heterogeneous data sources has grown in importance within the last years. One of the main reasons is the increasing availability of web-based data sources. Even within a single organization, data from disparate sources must be integrated. Our approach to solve this problem is based on Web ontologies. As we focus on the integration of multimedia assets, our base ontology is the MPEG-7 OWL ontology. 
When multimedia metadata based on different schemes has to be integrated, the XML Schemas are first mapped to OWL. Once this first step has been done, these schemas are easily integrated into the ontological framework using OWL semantic relations for equivalence and inclusion: subClassOf, subPropertyOf, equivalentClass, equivalentProperty, sameIndividualAs, etc. These relationships capture the semantics of the data integration. Then, once metadata is incorporated into the system and semantically-decorated, the integration is automatically performed by applying inference.

Our study on metadata integration is based on three different schemas: MusicBrainz schema, Foafing the Music ontology and a music vocabulary to describe performances. MusicBrainz is a community music metadatabase that attempts to create a comprehensive music information site. MusicBrainz schema is written in $\mathrm{RDF}$, and describes all the tracks, albums and artists available in their music repository. Their mappings to the MPEG-7 OWL ontology are shown in Table 3.

\section{Table 3. MusicBrainz to MPEG-7 OWL ontology mappings}

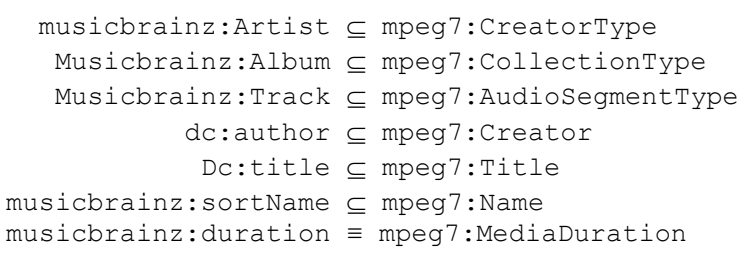

The Foafing the Music ontology describes (low-level) content-based descriptors extracted automatically from the audio itself. The mappings of this schema to the MPEG-7 OWL ontology are summarized in Table 4. An artist is defined as a subclass of the MPEG-7 Creator type, a track is defined as a subclass of the MPEG-7 AudioSegment and the audio Descriptor class describes the content-based properties of a track. This descriptor is linked with the MPEG-7 AudioDS type. Thus, all Foafing the Music descriptors' subclasses inherit the properties from the MPEG-7 Audio descriptor scheme. To characterize the descriptors related with the tonality of a song, Foafing the Music ontology defines some properties, such as mode and key. Finally, the ontology defines rhythm descriptors to describe the rhythm component of a track, e.g. meter and tempo.

The last of the three schemas, a music vocabulary to describe performances, is linked, as well with the MPEG-7 OWL (see Table 5). This schema models -for example, in the classical music world- a concert with the conductor, performers, the whole program, time schedule, etc. The most general class related with a music piece is the Musical_Unit, from which all types of performances are derived (e.g. an opera performance, a symphony, a movement of the symphony, etc.).

Table 4. Foafing the Music ontology to MPEG-7 OWL ontology mappings

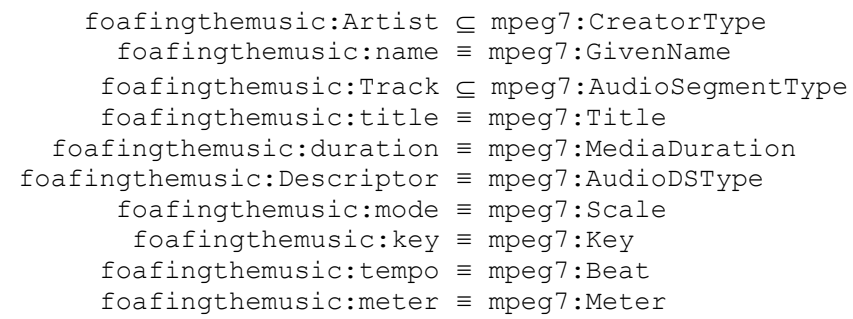

Decomposition of a musical unit is achieved by defining its sections, and we link it with the MPEG-7 AudioSegment. Finally, there is an Artist class, the superclass for all the agents of the performances, e.g. 
director, musician, singer, etc. Therefore, we link the Artist class with MPEG-7 OWL and, automatically (transitivity property of rdfs: subClassOf) all the subclasses are linked with the MPEG-7 OWL ontology.

\section{Table 5. Music Vocabulary ontology to MPEG-7 OWL ontology mappings}

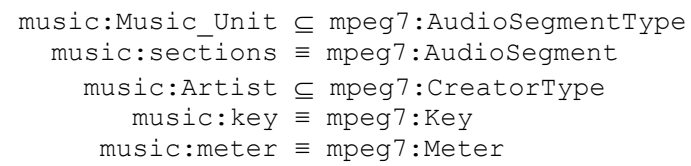

Once these mappings are done, all the multimedia assets are integrated into the ontological framework; that is the MPEG-7 OWL linked with all the schemas. Now, querying the system for audio segments will retrieve information from all the different sources, transparently to the user.

\subsection{Semantic Retrieval of Music Metadata}

Retrieving multimedia assets in the proposed architecture can be easily achieved by using semantic query languages like the SPARQL Query Language (Prud'hommeaux and Seaborne, 2007). SPARQL can take profit from the semantics made explicit by the XSD2OWL and XML2RDF mappings. It can, as well, exploiting the results of semantic rules for metadata integration in order to retrieve all the related multimedia information for a given query. In our case, SPARQL queries use the MPEG-7 OWL ontology "vocabulary" in order to integrate all data source. Using the mappings explained in the previous section, an SPARQL query can acquire information from MusicBrainz, Foafing the Music, the classical music ontology, etc.

A typical scenario that shows the usefulness of the architecture proposed could be the following: an Internet crawler is looking for audio data (let's assume that is searching for MP3 files) and it downloads all the files. Getting editorial and related information for these audio files can be achieved reading the information stored in the ID3 tag. Unfortunately, sometimes there is no basic editorial information like the title of the track, or the performer.

However, content-based low-level descriptors can be computed for these files, including its MusicBrainz fingerprint, a string that uniquely identifies each audio file based on its content. The example in Table 6 shows an RDF/N3 description for a track with the calculated tempo and fingerprint.

Table 6. Content-based metadata, tempo and fingerprint

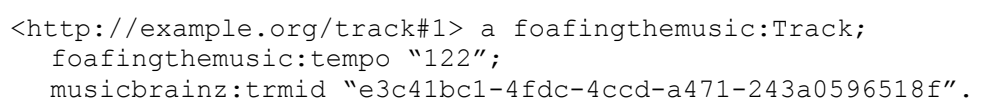

On the other hand, MusicBrainz database has the editorial metadata -as well as the fingerprint already calculated- for more than 3 millions of tracks. For example, the RDF description of the song “Blowin' in the wind" composed by Bob Dylan in Table 7.

Table 7. Editorial metadata, title and author, plus fingerprint

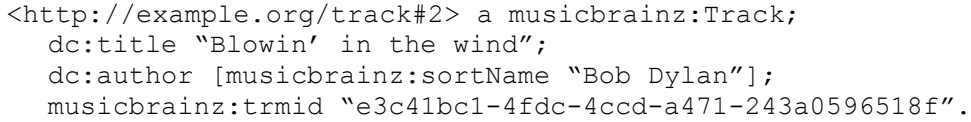


A closer look to both examples should highlight that the two resources are sharing the same $\mathrm{Mu}$ sicBrainz's fingerprint. Therefore, it is clear that, using a simple rule (1), one can assert that both audio files are actually the same file, that is to say the same instance in terms of OWL, owl:sameIndividualAs.

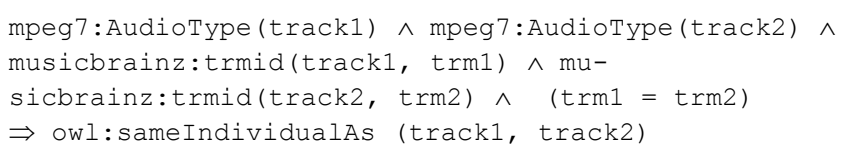

From now on, we have merged the metadata from both sources and we have deduced that the metadata related with both tracks is in fact referring to the same track. This data integration (at the instance level) is very powerful as it can combine and merge context-based data (editorial, cultural, etc.) with content-based data (extracted from the audio itself).

Finally, issuing SPARQL query that searches for all the songs composed by Bob Dylan that have a fast tempo, retrieves a list of songs, including "Blowin' in the wind”. Moreover, there is no need for metadata provenance awareness at the end-user level. As the example in Table 8 shows, all query terms are referred only to the MPEG-7 ontology namespace.

Table 8. SPARQL query for integrated metadata retrieval

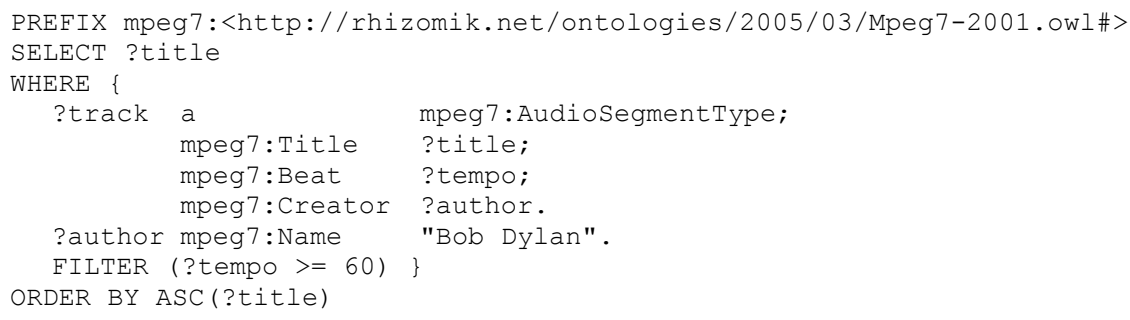

\section{An Integrated Ontological Infrastructure for the Semantic Description of Multimedia Content}

In this section we present the DS-MIRF Ontological Infrastructure, an integrated ontological infrastructure for the semantic description of multimedia content that allows for the systematic integration of domain knowledge in the MPEG-7 semantics. This infrastructure was developed in the context of the DS-MIRF framework (Tsinaraki, Polydoros and Christodoulakis 2007), which facilitates the development of knowledge-based multimedia content services based on the MPEG-7/21 standards. The DS-MIRF ontological infrastructure can support different usage scenarios, which fall in two main categories:

- The usage scenarios where the DS-MIRF ontological infrastructure is used in order to guide MPEG-7 based semantic multimedia content annotation and/or semantic multimedia service provision on top of an OWL/RDF repository. In this case, the OWL/RDF semantic multimedia annotations that are produced (manually, automatically or semi-automatically), possibly after being enriched through the application of rule-based reasoning, are stored in the repository.

- The usage scenarios where the DS-MIRF ontological infrastructure is used in order to guide MPEG-7 based semantic multimedia content annotation and/or semantic multimedia service provision on top of a pure MPEG-7 repository. In this case, the OWL/RDF semantic multimedia annotations that are produced, possibly after being enriched through reasoning, are transformed into pure MPEG-7 descriptions and are then stored in the repository. This category of usage scenarios is extremely useful both for groups using 
pure MPEG-7 and for groups sharing pure MPEG-7 descriptions with their partners. Full support for this category of usage scenarios is provided by the DS-MIRF framework.

The ontological infrastructure of the DS-MIRF framework (depicted in Fig. 7) includes an $O W L-D L U p$ per Ontology, OWL-DL Application Ontologies and OWL-DL Domain Ontologies.

The OWL-DL Upper Ontology fully captures the semantics of the MPEG-7 MDS and the MPEG-21 DIA Architecture (ISO/IEC 2004) and the parts of the MPEG-7 Visual and Audio that are necessary for the complete representation of the MPEG-7 MDS. This ontology includes the MPEG-7 OWL-DL ontology described in section 4, extended with the MPEG-21 DIA Architecture semantics in order to better support multimedia content personalisation and adaptation.

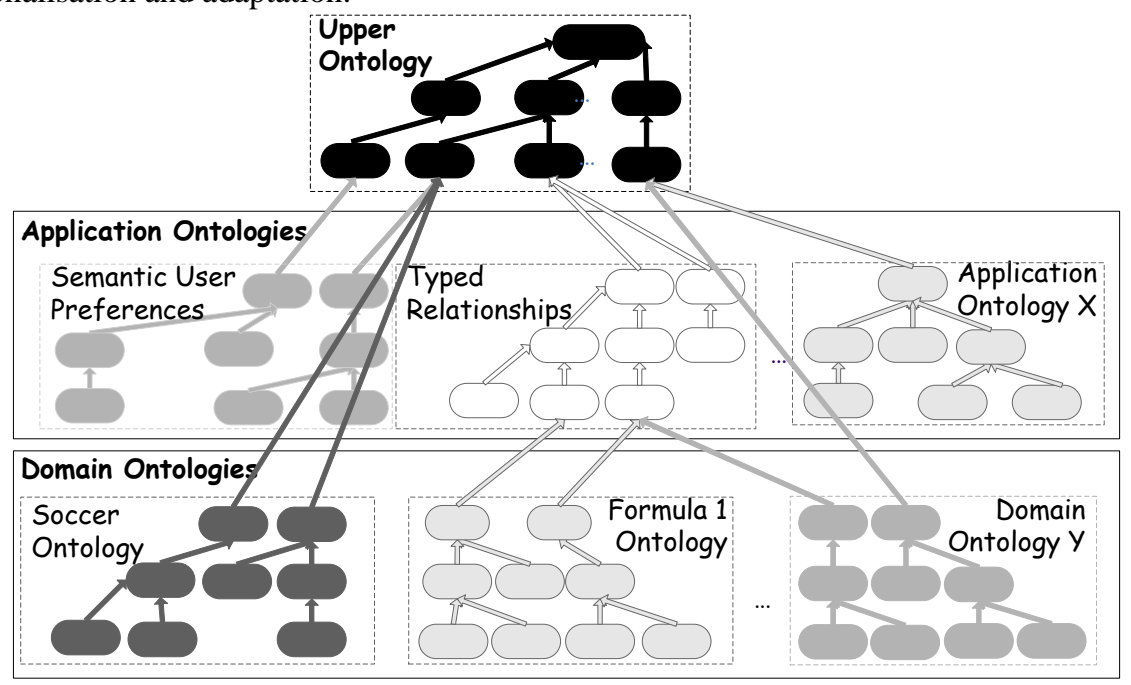

Fig. 7. The Ontological Infrastructure of the DS-MIRF Framework

The OWL Application Ontologies either enhance, using OWL-DL syntax, the semantics of MPEG-7/21 so that the users find it easier to use MPEG-7/21 or are able to use advanced multimedia content services that cannot be directly supported by the MPEG-7/21. The application ontologies provide general-purpose constructs that either are not available in MPEG-7/21 (like, for example, semantic user preferences) or are implied in the text of MPEG-7/21 but missing in their syntax (like, for example, typed relationships).

The Domain Ontologies systematically extend the Upper ontology and the Application ontologies with domain knowledge (like, for example, sports ontologies that extend the abstract semantic description capabilities of the MPEG-7 MDS).

In the rest of this section we present the application ontologies that have been already integrated in the DS-MIRF ontological infrastructure (in subsection 7.1), the methodology followed for domain knowledge representation in the form of OWL domain ontologies and their integration with the MPEG-7 semantics (in subsection 7.2) and the DS-MIRF framework and the support it provides for pure MPEG-7 applications (in subsection 7.3).

\subsection{Application Ontologies}

We outline in this subsection the application ontologies that have already been integrated in the DS-MIRF framework. These include: (a) A Typed Relationship application ontology, which extends the MPEG-7 MDS in order to allow the full and systematic representation of typed relationships that are literally described in the 
MPEG-7 MDS text but their features are not fully captured in the MPEG-7 MDS syntax; and (b) A Semantic User Preference application ontology that supports the semantic-based description of the desired multimedia content, which is not allowed in the MPEG-7 user preferences. The application ontologies are described in the following paragraphs.

Typed Relationship Application Ontology. The Typed Relationship application ontology assists the semantic multimedia content description. It extends the MPEG-7 MDS in order to allow the full and systematic representation of typed relationships that are literally described in the MPEG-7 MDS text but their features are not fully represented in the MPEG-7 MDS syntax. The typed relationship ontology is an application ontology that can greatly facilitate application development by the users in the large majority of the cases. The users are not forced to use this ontology, but if they do so, the definition of relationships in MPEG-7 metadata descriptions becomes much easier.

The semantics of the typed relationships are partially covered in the MPEG-7 MDS syntax in the GraphReletion, SpatialRelation, SemanticRelation, BaseRelation and TemporalRelation classification schemes. The representation of the relationship types in the form of classification scheme terms does not allow expressing formally if a relationship is directed and, if so, which is its inverse relationship; this information is available only in the textual description of the relationship type.

The typed relationship ontology, depicted in Fig. 8, extends the Upper ontology with an OWL class hierarchy rooted in the TypedRelationType (which is a subclass of the RelationType class of the Upper ontology that represents relationships). The direct subclasses of TypedRelationType are homonyms of the classification schemes where the relationship types are defined. Each of the subclasses of TypedRelationType has a number of subclasses, which correspond to the relationship types defined in the homonym classification scheme, together with the information literally described about them in the MPEG-7 MDS text. This information includes the type of the relationship, if it is directed or not and, in the later case, its inverse relationship. The annotator that uses the typed relationship application ontology does not have to be aware of the textual description of the MPEG-7 MDS, since all the information is captured in the ontology.

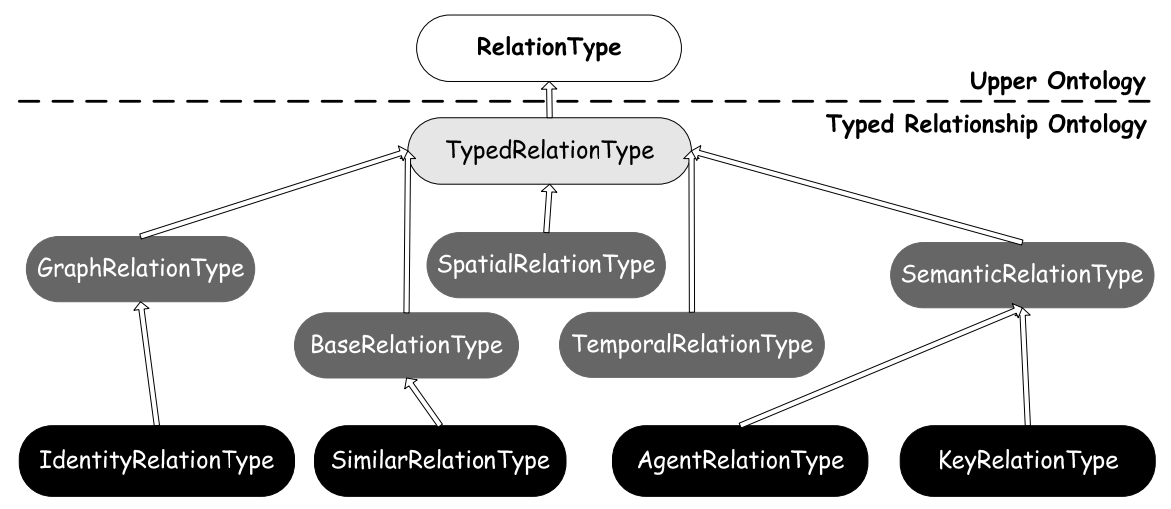

Fig. 8. The Typed Relationship Ontology

The OWL classes of the typed relationship ontology formally capture all the information about the typed relationships that exist in the MPEG-7 MDS text. In fact, they express formally the semantics that exist in the textual descriptions of the different relationship types (for example, that the before relationship is directed and that the after relationship is its inverse).

Semantic User Preference Application Ontology. The Semantic User Preference application ontology allows the semantic-based description of the desired multimedia content in the user preferences. Such an extension of the MPEG-7/21 is needed, because the MPEG-7/21 user preference descriptions allow keyword- 
only descriptions of the semantics of the preferred content. As an example, consider a user who wishes to receive all the images that contain a teacher who gives a book to a student, as soon as such images are available. The current MPEG-7 search and filtering preference descriptions allow the users to describe the desired images using the keywords teacher, student, gives and book. These user preference descriptions will provide, together with the images that contain a teacher who gives a book to a student, images that contain a student who gives a book to a teacher.

The application ontology is based on the semantic user preference model proposed in (Tsinaraki and Christodoulakis 2007), which is also compatible with the MP7QL and allows for the explicit specification of the boolean operators to be used in the different phases of multimedia content search and filtering. The semantic user preferences structured according to this model allow the accurate expression of the user preferences of a user who wishes to receive all the images that contain a teacher who gives a book to a student.

\subsection{Domain Knowledge Representation and Integration with the MPEG-7}

The multimedia content description approaches, which have been implemented in MPEG-7 and have been described in section 2 are general-purpose and can be applied in any domain. In particular, the generalpurpose semantic description capabilities of the MPEG-7 distinguish only events, agents (people, person groups and organisations), places, states, times, objects and concepts. On the other hand, the systematic integration of domain knowledge in the multimedia content descriptions has been shown to enhance the retrieval effectiveness of the multimedia content retrieval and filtering services built on top of them. We outline in this subsection a methodology for domain knowledge representation in OWL and its integration with the MPEG7 semantics (Tsinaraki, Polydoros and Christodoulakis 2007; Tsinaraki, Polydoros and Christodoulakis 2004a). This methodology has been developed in the DS-MIRF framework for the definition and integration of domain ontologies in the DS-MIRF ontological infrastructure. Thanks to this methodology, OWL/RDF multimedia content descriptions can be defined that are structured according to the MPEG-7 semantics and are also enhanced with domain knowledge.

According to this methodology, the domain-specific entities are represented as domain ontology classes. These classes are (direct or indirect) subclasses of the OWL classes that represent the subtypes of SemanticBaseType (EventType, ObjectType, AgentObjectType, SemanticPlaceType, SemanticTimeType, SemanticStateType and ConceptType) in the Upper OWL-DL ontology defined in (Tsinaraki, Polydoros and Christodoulakis 2004b). This way, the knowledge captured in the domain ontologies is integrated with the MPEG-7 semantic model. As an example, the Article class (shown in Fig. 9), which represents the articles, should be defined as a subclass of the ObjectType class.

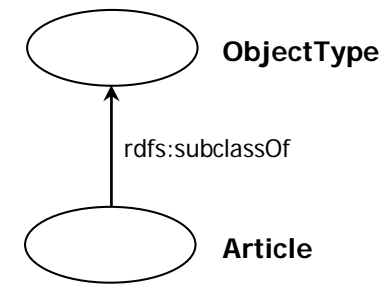

Fig. 9. RDF Graph showing the Article Class, which Represents Articles

Features that are not present in the Upper ontology class are represented as additional object or datatype properties in its domain-specific subclass. For example, the number of pages of an article should be represented as a datatype property of non-negative integer type in the domain of the Article class. 
Additional constraints may be applied on the properties inherited from the ancestor classes, in order to guide the indexers to produce valid metadata (for example, the author of an article should have a name).

In addition, properties may be defined that permit attaching relationships to the allowed domain-specific entities only (for example, only persons are allowed to be related with articles as authors). These properties are subproperties of the Relation property of the SemanticBaseType class, which links semantic entities with relationships. The properties have as domain the union of the classes to which belong individuals that are capable of being sources of a typed relationship and the typed relationship class as range. The inverse property of the one defined previously is defined in the domain of the classes the individuals of which are capable of being targets of the typed relationship.

The methodology described above can be also used in order to integrate existing OWL domain ontologies in the MPEG-7 semantics. It has been tested in the DS-MIRF framework through the definition of domain ontologies for soccer and Formula 1 and their integration with the DS-MIRF ontological infrastructure.

\subsection{The DS-MIRF Framework}

The architecture of the DS-MIRF framework and the information flow between its components are depicted in Fig. 10.

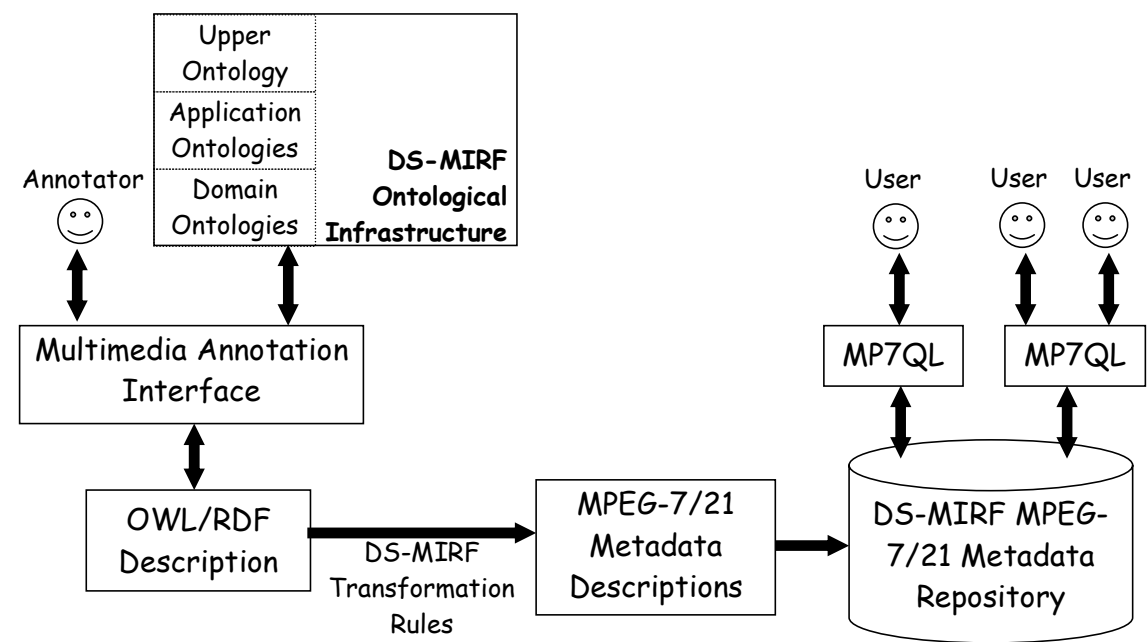

Fig. 10. The DS-MIRF Framework - Architecture and Information Flow

The multimedia content annotator is a special type of user in the DS-MIRF framework that is responsible for the semantic annotation of multimedia documents. He uses a multimedia annotation interface that makes use of the ontological infrastructure of the DS-MIRF framework in order to support ontology-based semantic annotation of the multimedia content. The DS-MIRF framework ontologies are expressed in OWL, thus the result of the annotation process is an OWL description of the multimedia content. The OWL descriptions are then transformed, using the DS-MIRF transformation rules to standard MPEG-7/21 metadata descriptions. The MPEG-7/21 metadata are stored in the DS-MIRF MPEG-7/21 Metadata Repository, which is accessed by the end-users through application interfaces that are based on the MPEG-7 Query Language (MP7QL) (Tsinaraki and Christodoulakis 2007), a query language that has been developed in the context of the DSMIRF framework for querying MPEG-7 multimedia descriptions. 
In the following paragraphs we will focus on the MP7QL query language and on the support for interoperation with applications using pure MPEG-7.

The MP7QL Query Language. The MP7QL query language has the MPEG-7 as data model and allows for querying every aspect of an MPEG-7 multimedia content description, including semantics, low-level visual features and media-related aspects. It also allows for the exploitation of domain knowledge encoded using pure MPEG-7 constructs. In addition, it allows the explicit specification of boolean operators and/or preference values. The MP7QL queries may utilize the users' Filtering and Search Preferences (FASP) and Usage History as context, thus allowing for personalized multimedia content retrieval. The MP7QL has been expressed both in XML Schema and OWL based syntax, in order to be capable for all the usage scenarios and working environments. The XML Schema based syntax of the MP7QL is used in the current implementation of the DS-MIRF framework.

General-purpose languages, like the XQuery (Simeon et. al. 2007) in the pure MPEG-7 environment and the SPARQL in the Semantic Web environment, do not take into account the following peculiarities of the MPEG-7 description elements: (a) The MPEG-7 semantic model is expressed in an involved way; (b) The domain knowledge integrated in the semantic MPEG-7 descriptions is expressed in the document level; and (c) The low-level visual and audio features should be evaluated using specialized functions. Thus, in order to fully exploit the semantics of the MPEG-7 descriptions, a query language for querying MPEG-7 descriptions is needed, with clear, MPEG-7 specific semantics (instead of the generic semantics of the XQuery and SPARQL). These semantics will also allow the optimizers to effectively perform consistency checking and first-level optimization. The MP7QL fulfils the requirement for MPEG-7 semantics, as it has the MPEG-7 as data model.

Support for Interoperation with Applications using Pure MPEG-7. Interoperation of the multimedia content descriptions with applications using pure MPEG-7 is achieved through the DS-MIRF transformation rules that allow the transformation of domain ontologies and semantic content descriptions to valid MPEG-7 descriptions. In particular, they allow the transformation of: (a) Domain ontologies defined according to the methodology described in (Tsinaraki, Polydoros, Kazasis and Christodoulakis 2005) into abstract MPEG7/21 semantic descriptions; (b) OWL individuals that belong to the domain ontology classes into MPEG-7/21 semantic descriptions. The produced descriptions are valid MPEG-7/21 (parts of) documents.

During the metadata transformation from OWL to MPEG-7/21, the individuals representing MPEG-7/21 constructs are transformed into XML elements. The object properties are transformed into elements and the datatype properties are transformed into the constructs they represent in the original MPEG-7/21 schemas (attributes, elements or simple values). In order to produce valid MPEG-7/21 descriptions, information regarding the MPEG-7/21 XML element order, the default values and the original MPEG-7/21 representation of the datatype properties is needed. This information is kept in a transformation rule ontology and is utilised during both ontology and metadata transformations, as shown in Fig. 11.

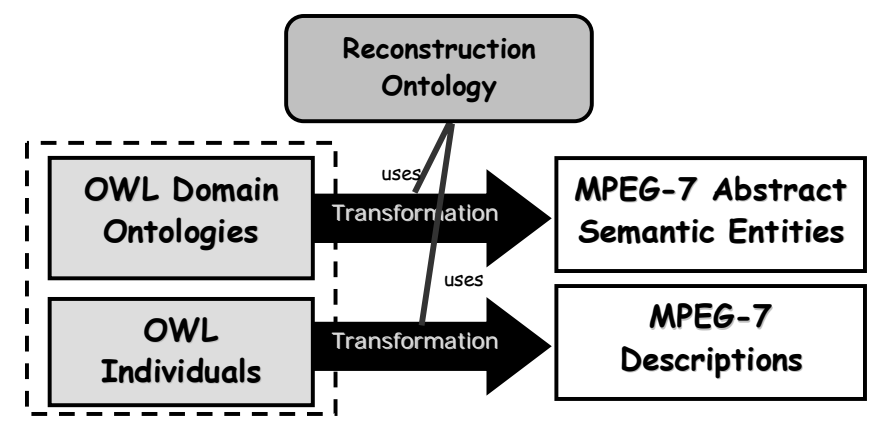

Fig. 11. OWL-MPEG-7 Transformations in the DS-MIRF Framework 
The classes of the OWL domain ontologies and the OWL individuals belonging to them are both transformed into instances of the subtypes of SemanticBaseType. This way, the domain knowledge is represented in a way compatible with the domain knowledge expressed according to the methodology presented in (Tsinaraki, Polydoros, Kazasis and Christodoulakis 2005). The AbstractionLevel element of the SemanticBaseType and the MPEG-7 semantic relationships are used to capture the ontology semantics. An abstract semantic entity that represents a domain-specific class has a non-zero AbstractionLevel.Dimension and is related with the semantic entities that represent its subclasses through: (a) A relationship of type generalizes, which has as source the semantic entity that represents the class and as target the semantic entity that represents the subclass; and (b) A relationship of type specializes, which has as source the semantic entity that represents the subclass and as target the semantic entity that represents the class . In addition, an abstract semantic entity that represents a class is related with each of the semantic entities representing the class individuals through pairs of exemplifies/exemplifiedBy relationships.

The datatype properties of the classes of the domain ontologies are transformed into Property elements and the object properties into pairs of property/propertyOf relationships.

The transformations outlined above allow the representation of the OWL class hierarchy and the preservation of the class properties. Several OWL axioms, especially the restrictions and the set operations cannot be expressed in the pure MPEG-7 syntax. This feature does not allow using reasoning on top of the pure MPEG7 descriptions, but it allows the systematic use of the domain knowledge that is expressed using MPEG-7 constructs. As a consequence, queries of the form "Give me the multimedia objects that show a teacher who gives a book to a student” can be expressed accurately, instead of searching in the textual parts of the MPEG7 description elements (including the semantic ones) for the keywords teacher, student, gives and book. The latter query is also ambiguous, as it will also return the multimedia objects where a student gives a book to a teacher. In addition, it may evaluate as teachers semantic entities that represent people that have worked as teachers for a while and the keyword teacher exists in the textual annotation of the semantic entities.

Another approach for domain knowledge representation is the definition of subtypes of the MPEG-7 types that represent semantic entities in order to represent domain-specific classes. The advantage of the utilization of abstract semantic entities instead of subtypes of the semantic entity types for the representation of domain-specific classes is that this way full compatibility with MPEG-7 is maintained so that all the tools and the applications that use pure MPEG-7 still work transparently with the produced MPEG-7 descriptions.

The ontological infrastructure of the DS-MIRF framework and the mechanisms that have been developed in the context of DS-MIRF for the support of interoperability between OWL domain ontologies and MPEG7/21 support the semantic multimedia content description, which in turn allows providing advanced (semantic) multimedia content services. In particular, advanced retrieval services can be supported on top of the semantic multimedia annotations, which allow more accurate multimedia content retrieval. Accurate retrieval results in the better support of advanced services built on top of it, like filtering, content-based personalisation etc. Such services are offered in the DS-MIRF framework based on the MP7QL language that supports personalised semantic retrieval and filtering on top of MPEG-7 multimedia content descriptions.

\section{Conclusions}

In this chapter we have introduced the need for representing the MPEG-7 constructs using the Semantic Web languages. First, we presented in section 2 the MPEG-7 standard and described the well-accepted general purpose approaches for multimedia content description that are supported by the MPEG-7 standard and their implementation in MPEG-7. Then, we have presented the Semantic Web languages in section 3. The research efforts towards the expression of the MPEG-7 using the Semantic Web languages have been outlined in section 4, followed by the mapping of the MPEG-7 constructs to OWL constructs in section 5. The application of two of the MPEG-7 ontologies in real application environments have been presented next: (a) A case study 
for the music domain has been presented in section 6, which has introduced the problems of annotating multimedia assets, integrating data from different sources, and retrieving music related descriptors; and (b) An integrated ontological infrastructure for the semantic description of multimedia content, which allows for combining the general-purpose MPEG-7 constructs with domain and application specific knowledge has been described in section 7 .

Both application scenarios show the benefits of the MPEG-7 formal semantics. MPEG-7 is a big standard, difficult to deal with but the availability of some formal semantics facilitates the development of more advanced tools capable of dealing with its complexity.

\section{References}

The AceMedia project (2007) Harmonization of Multimedia Ontologies activity. http://www.acemedia.org/aceMedia/reference/multimedia_ontology/index.html

Antoniou, G. and Harmelen, F. van. A Semantic Web Primer. The MIT Press, April 2004.

Amann, B., Beer, C., Fundulak, I. and Scholl, M. (2002) Ontology-Based Integration of XML Web Resources. In Proceedings of the 1st International Semantic Web Conference (ISWC 2002).

Berners-Lee, T. Weaving the Web. Texere Publishing Ltd., November 1999.

Berners-Lee, T, Hendler, J., and Lassila, O.. The semantic web. Scientific American, May 2001.

Brickley, D. and Guha, R. V. (eds.) (2004) RDF Vocabulary Description Language 1.0: RDF Schema. W3C Recommendation.

Celma O., Dasiopoulou S., Hausenblas M., Little S., Tsinaraki C. and Troncy R. (2007) MPEG-7 and the Semantic Web. W3C Multimedia Semantics Incubator Group (MMSEM-XG) Deliverable, http://www.w3.org/2005/Incubator/mmsem/XGR-mpeg7/.

Cruz, I., Xiao, H. and Hsu, F. (2004) An Ontology-based Framework for XML Semantic Integration. In Proceedings of the Eighth International Database Engineering and Applications Symposium. Coimbra, Portugal.

Fallside, D. (2001) XML Schema Part 0: Primer. W3C Recommendation.

García, R. and Celma, O. (2005) Semantic Integration and Retrieval of Multimedia Metadata. In the proc. of the Knowledge Markup and Semantic Annotation Workshop, Semannot'05.

García, R. (2006) A Semantic Web Approach to Digital Rights Management. PhD thesis, Technologies Department, Universitat Pompeu Fabra. http://rhizomik.net/ roberto/thesis

García, R. and Gil, R. (2007) Facilitating Business Interoperability from the Semantic Web. In Proc. 10th International Conference on Business Information Systems, BIS'07. Springer-Verlag, Lecture Notes in Computer Science, (4439):220-232.

García, R.; Gil, R. and Delgado, J. (2007) A Web Ontologies Framework for Digital Rights Management. Journal of Artificial Intelligence and Law, 15(2):online first

Gruber, T. R. Towards principles for the design of ontologies used for knowledge sharing. In N. Guarino and R. Poli, editors, Formal Ontology in Conceptual Analysis and Knowledge Representation, Deventer, The Netherlands, 1993. Kluwer Academic Publishers.

Halevy, A., Ives, Z., Mork, P. and Tatarinov, I. (2003) Piazza: Data Management Infrastructure for Semantic Web Applications. In Proceedings of the 12th World Wide Web Conference. Budapest, Hungary, 556-567.

Klein, M.C.A. (2002) Interpreting xml documents via an rdf schema ontology. In Proceedings of the 13th International Workshop on Database and Expert Systems Applications (DEXA 2002).

McGuinness, D. L. and van Harmelen, F. (eds.) (2004) OWL Web Ontology Language: Overview. W3C Recommendation.

McGuinness, D. L., Fikes, R., Hendler, J. and Stein. L. A. (2002) DAML+OIL: An Ontology Language for the Semantic Web. In IEEE Intelligent Systems, Sep.-Oct. 2002, 17(5):72-80

Hunter, J., (1999) A proposal for an mpeg-7 description definition language. Technical report, MPEG-7 AHG Test and Evaluation Meeting, Lancaster, UK

Hunter, J., (2001) Adding Multimedia to the Semantic Web - Building an MPEG-7 Ontology. In the proc. of the International Semantic Web Working Symposium (SWWS), 2001.

ISO MPEG Group MPEG-7 (Multimedia Content Description Interface). 
ISO/IEC (2001a) 15938-3:2001: Information Technology - Multimedia content description interface - Part 3 visual. Version 1.

ISO/IEC (2001b) 15938-3:2001: Information Technology - Multimedia content description interface - Part 4 audio. Version 1.

ISO/IEC (2003) 15938-5:2003: Information Technology - Multimedia content description interface - Part 5: Multimedia description schemes. First Edition.

ISO/IEC (2004) 21000-7:2004, Information Technology - Multimedia Framework (MPEG-21) - Part 7: Digital Item Adaptation, 2004.

Lakshmanan, L. and Sadri, F. (2003) Interoperability on XML Data. In Proceedings of the 2nd International Semantic Web Conference (ICSW 03).

Manola, F. and Milles, E. (eds.) (2004) RDF Primer. W3C Recommendation.

Patel-Schneider, P. and Simeon, J. (2002) The Yin/Yang web: XML syntax and RDF semantics. In Proceedings of the 11th International World Wide Web Conference (WWW02), Honolulu, Hawaii, USA, 443-453.

Prud'hommeaux R., Seaborne A. (2007). SPARQL Query Language for RDF. W3C Working Draft, 26 March 2007. (http://www.w3.org/TR/rdf-sparql-query/).

Salembier P. Manjunath, B. S. and T. Sikora (2002). Introduction to MPEG 7: Multimedia Content Description Language. Ed. Wiley, 2002.

Shadbolt, N. , Berners-Lee, T., and Hall, W. The semantic web revisited. IEEE Intelligent Systems, 21(3):96-101, 2006.

Siméon, J., Chamberlin, D., Fernández, M., Boag, S., Florescu, D., Robie, J. (Eds) (2007) XQuery 1.0: An XML Query Language. W3C Recommendation, 23 Jan. 2007. (http://www.w3.org/TR/xquery/)

Simou, N., Tzouvaras, V., Avrithis, Y., Stamou, G. and Kollias, S. (2005) A Visual Descriptor Ontology for Multimedia Reasoning. In the proc. of the Workshop on Image Analysis for Multimedia Interactive Services (WIAMIS) 2005.

Tous, R., García, R., Rodríguez, E. and Delgado, J. (2005) Arquitecture of a Semantic XPath Processor. Application to Digital Rights Management (DRM). In the Proceedings of E-Commerce and Web Technologies: 6th International Conference, EC-Web 2005. Springer-Verlag, LNCS (3590):1-10

Tsinaraki, C. and Christodoulakis, S. (2007) An MPEG-7 Query Language and a User Preference Model that allow Semantic Retrieval and Filtering of Multimedia Content. ACM-Springer Multimedia Systems Journal, in Special Issue on Semantic Multimedia Adaptation and Personalization, 2007 (to appear)

Tsinaraki, C., Polydoros, P. and Christodoulakis, S. (2007) Interoperability support between MPEG-7/21 and OWL in DSMIRF. Transactions on Knowledge and Data Engineering (TKDE), Special Issue on the Semantic Web Era, pp. 219232.

Tsinaraki, C., Polydoros, P. and Christodoulakis, S. (2004a) Integration of OWL ontologies in MPEG-7 and TV-Anytime compliant Semantic Indexing. In the proc. of the $16^{\text {th }}$ International Conference on Advanced Information Systems Engineering (CAISE), pp. 398-413.

Tsinaraki, C., Polydoros, P. and Christodoulakis, S. (2004b) Interoperability support for Ontology-based Video Retrieval Applications. In the proc. of the Conference on Image and Video Retrieval (CIVR) 2004, pp. 582-591.

Tsinaraki, C., Polydoros, P. Kazasis, F. and Christodoulakis, S. (2005) Ontology-based Semantic Indexing for MPEG-7 and TV-Anytime Audiovisual Content. In Multimedia Tools and Application Journal (MTAP), Special Issue of on Video Segmentation for Semantic Annotation and Transcoding, 26, pp. 299-325, August 2005.

Tzouvaras, V. and Pan, J. (2007) Multimedia Annotation Interoperability Framework. W3C Multimedia Semantics Incubator Group (MMSEM-XG) Deliverable, http://www.w3.org/2005/Incubator/mmsem/wiki/Semantic_Interoperability. 